\title{
Fixing Up Waco, Texas: Populäre Religion, das Sentimentale und die Refiguration von Räumen
}

\author{
Silke Steets $\mathbb{D}$
}

Eingegangen: 20. September 2021 / Überarbeitet: 13. Oktober 2021 / Angenommen: 18. Oktober 2021 / Online publiziert: 28. Oktober 2021

(C) Der/die Autor(en) 2021

Zusammenfassung Der Text untersucht aktuelle religiöse Transformationsprozesse in der texanischen Stadt Waco als räumlich-kommunikative Refiguration. Ausgangspunkt ist der durchschlagende TV-Erfolg der Hausrenovierungsshow Fixer Upper, die in Waco spielt, in bislang fünf Staffeln bis zu 75 Millionen Zuschauer:innen fand und vor Ort einen Tourismusboom auslöste, der zu einem erstaunlichen Imagewandel der Stadt beitrug. Auf der Grundlage ethnographischer Beobachtungen und Interviewdaten sowie einer umfassenden Dokumentenanalyse rekonstruiert der Text schrittweise eine neue popkulturell-touristische Kommunikationsform des Religiösen, die unterschiedliche Formate annehmen kann - von der TV Show bis zur Stadtführung. Geprägt ist diese Kommunikationsform erstens durch ein evangelikales Erneuerungsnarrativ, zweitens durch die rituelle Herstellung und das materielle Wirklichwerden einer sentimentalen Neuordnung von Räumen und drittens durch die Ästhetisierung der evangelikalen Missionierungskommunikation. Sie setzt einen Wandlungsprozess in Gang, bei dem sich Raum und Religion wechselseitig bedingen: Einerseits wird der Raum zum zentralen Medium der religiösen Kommunikation, andererseits fungiert die Religion als identitätsstiftender Bezugspunkt spätmoderner Raumkonstruktionen. Die Analyse macht schließlich deutlich, dass sich im Raum ein Ringen um Aufmerksamkeit und Macht entfaltet, das sich als Resultat einer Spannung zwischen Bahnenraum und Ort und damit als Refiguration von Räumen deuten lässt.

Schlüsselwörter Evangelikalismus $\cdot$ Raum $\cdot$ Tourismus $\cdot$ Sentimentalität

Silke Steets $(\bowtie)$

Institut für Soziologie, Friedrich-Alexander-Universität Erlangen-Nürnberg,

Kochstraße 4, 91054 Erlangen, Deutschland

E-Mail: silke.steets@fau.de 


\section{Fixing Up Waco, Texas: Popular religion, sentimentality and the refiguration of spaces}

Abstract This article examines the current religious transformation processes in the Texas city of Waco as a spatial-communicative refiguration. The starting point is the resounding success of the TV house renovation show Fixer Upper, which is set in Waco, attracted up to 75 million viewers in five seasons so far, and triggered a local tourism boom that contributed to an astonishing image transformation of the city. Drawing on ethnographic observations and interview data, as well as extensive document analysis, the text reconstructs a new form of popular religion that uses pop cultural and tourist motifs and adopts a variety of formats, ranging from television shows to city tours. This new form of popular religion is characterized, first, by an evangelical renewal narrative, second, by the ritual staging and material implementation of a sentimental reorganization of spaces, and third, by the aestheticization of evangelical mission communication. It triggers a process of change in which space and religion are mutually dependent: On the one hand, space becomes the central medium of religious communication; on the other hand, religion functions as an identity-forming factor for late modern productions of space. Finally, the analysis shows that a struggle for attention and power unfolds in space that can be interpreted as the result of a tension between the spatial logic of trajectory space (Bahnenraum) and place (Ort) and thus as a refiguration of spaces.

Keywords Evangelicalism $\cdot$ Space $\cdot$ Tourism $\cdot$ Sentimentality

\section{Einleitung}

Ziel des vorliegenden Textes ist es, aktuelle religiöse Transformationsprozesse in der texanischen Stadt Waco als räumlich-kommunikative Refiguration zu beschreiben. In der imaginären Geographie der USA wurde Waco lange Zeit mit der „Waco Siege" assoziiert, der wochenlangen Belagerung und verunglückten Erstürmung des Geländes der Branch-Davidian-Sekte durch das FBI im Jahr 1993, bei der über achtzig Menschen starben. Seither stand Waco vor allem für religiösen Fanatismus im texanischen Hinterland. In den letzten Jahren aber erlebte die Stadt einen rasanten symbolischen wie räumlichen Wandel, der durch den Erfolg der TV-Show Fixer Upper ausgelöst wurde. In Fixer Upper renoviert ein sympathisches Ehepaar, Chip und Joanna Gaines, heruntergekommene Häuser in Waco und Umgebung und übergibt das Ergebnis am Ende einer jeden Folge an stets überwältigte neue Hauseigentümer ${ }^{1}$. Rund 75 Mio. Zuschauerinnen haben die jüngste Staffel von Fixer Upper (2018) allein in den USA gesehen (Magnolia Network 2020), und offenbar waren viele von der Show so begeistert, dass sie begannen nach Waco zu reisen, um sich die im Fernsehen bestaunten Häuser in echt und vor Ort anzuschauen. 2018 besuchten insgesamt 2,6 Mio. Menschen die Stadt, Tendenz (vor Corona) steigend (Petersen

\footnotetext{
1 Zur besseren Lesbarkeit verwende ich in diesem Text mal männliche, mal weibliche Sprachformen, womit jeweils ausdrücklich alle Geschlechter gemeint sind.
} 
2019). Den Tourismusboom aufgreifend realisierten die Gaines 2015 in Downtown Waco ein Gelände für Fixer Upper-Merchandising: Magnolia Market at the Silos, das zum Dreh- und Angelpunkt der Besucherströme wurde. Darüber hinaus entstanden allerlei neue Shops für Inneneinrichtung, Kulinarik, Haushaltswaren, Garten und Antikmöbel sowie Restaurants, Cafés und Food Trucks, die der Stadt eine neue Lebendigkeit und Attraktivität verleihen. Der Markt für private Vermietungen von Ferienwohnungen und -häusern, vor allem über die Plattform Airbnb, wuchs stetig, und neue Unternehmen für touristische Dienstleistungen wurden gegründet.

Im Folgenden werde ich zentrale Facetten dieses Wandels aus religions- sowie aus stadt- und raumsoziologischer Perspektive rekonstruieren und im Format einer „dichten Beschreibung“ (Geertz 1987) ein komplexes Bild dieses Wandels zeichnen. Auf eine kurze Darstellung von Geschichte und Lage der Stadt Waco (2) folgt eine erste religionssoziologische Annäherung an die Erzählstruktur von Fixer Upper (3). Es wird sich zeigen, dass in der Show konservative Geschlechterrollen eine Modernisierung erfahren, die Show Raum nicht nur materiell, sondern auch symbolisch neu ordnet und das zentrale Fixer Upper-Narrativ der Grundstruktur evangelikaler Konversionserzählungen folgt. Eine stadtsoziologische Perspektive einnehmend zeichne ich anschließend die symbolisch-räumlichen Effekte des Fixer Upper-Booms in Waco nach (4). Neben dem konflikthaften Wechselspiel von Ausbreitung (Kreativszene, Airbnbs, Orte cooler evangelikaler Urbanität) und Verdrängung (ärmere Bewohnerinnen, Nichtweiße, Nichtevangelikale) steht das Narrativ im Mittelpunkt, das die Stadtführungen von Waco Tours prägt, einem von vier jungen wiedergeborenen Christen 2016 gegründeten Touristikunternehmen, das seither über 55.000 Menschen durch Waco führte. Die Analyse macht deutlich, dass auch dieses Narrativ von einem evangelikalen Erneuerungsmotiv bestimmt ist - nun aber verpackt als aufregende Wiedergeburt der ganzen Stadt. Es entsteht eine neue Sozialform der Populären Religion, die weitgehend implizit bleibt und sich im Schnittfeld von Evangelikalismus, Medien, Tourismus, Sentimentalität und Raum entfaltet. Abschließend (5) bündele ich die Befunde, indem ich das Wechselspiel von Raum und Religion schrittweise abstrakter fasse und den Wandel Wacos als religiös induzierte Refiguration von Räumen beschreibe.

Empirisch basiert die Analyse auf zwei mehrtägigen Feldaufenthalten in Waco im Februar und Mai 2019, inklusive Airbnb-Übernachtung, Magnolia-Shopping und Teilnahmen an Waco Tours-Stadtführungen. Darüber hinaus habe ich mit Kate*2, einem Mitglied der liberalen unitarischen Gemeinde von Waco, ein rund neunzigminütiges leitfadengestütztes Interview geführt, in dem es um die Entwicklung der Stadt und um Kates* Leben jenseits des religiösen Mainstreams ging. Ergänzt wurde die Analyse durch eine ausführliche Dokumentenrecherche zu Fixer Upper sowie zu Geschichte und Wandel Wacos, die journalistische Texte, digitale stadtgeschichtliche Archive, Webseiten und Social Media-Kanäle umfasste. Wenn ich in diesem Text das Adjektiv ,evangelikal“ benutze, dann setze ich mich von der Ethnobezeich-

\footnotetext{
${ }^{2}$ Ich verwende Sternchen, um Personen zu kennzeichnen, die anonymisiert wurden. Dies trifft auf Interviewpartnerinnen und Tour-Guides zu, nicht aber auf Personen, deren öffentliches Auftreten ich deute. Beispiele für letztere sind die Protagonisten aus der TV-Show Fixer Upper oder die Inhaberinnen von Waco Tours.
} 
nung der untersuchten Gruppierungen ab, die sich selbst in der Regel als ,christlich“ beschreiben. Nach dem hier verwendeten Verständnis des Wortes teilen Evangelikale gewisse Glaubensauffassungen und religiöse Praktiken (Elwert et al. 2017): Sie zeichnet zum einen die persönliche und verbindliche Entscheidung für ein Leben mit Jesus Christus aus, was meist auf ein individuelles Konversionserlebnis beziehungsweise eine persönliche Wiedergeburt zurückgeführt wird und als der einzige Weg zu Heil und Erlösung gilt. Zum anderen eint sie ein nahezu wörtliches Verständnis der Bibel und ein eher figürliches Gottesverständnis, das heißt, Gott wird als (strenger) Freund imaginiert, der in der Lage ist, in die Kausalität der Alltagswelt einzugreifen und dort seine deutbaren Spuren zu hinterlassen. Aus diesen Vorstellungen leiten Evangelikale in der Regel eine besonders ausgeprägte Missionierungspraxis sowie moralisch und politisch streng konservative Haltungen ab.

\section{Waco - eine kurze Geschichte der Stadt}

Die Stadt Waco liegt im Zentrum des US-Bundesstaates Texas am Brazos River, etwa auf halber Strecke zwischen der Metropolregion Dallas-Fort Worth im Norden und der Bundeshauptstadt Austin im Süden. Gegründet 1849 von europäischen Einwanderern auf den Überresten einer Siedlung der Wichita-Indianerinnen umfasst sie heute knapp 140.000 Einwohner (United States Census Bureau 2020). Nach der Stadtgründung wuchs die Bevölkerung rasch an. Waco wurde zum Verwaltungssitz des 1850 geformten McLennan County; wirtschaftlich dominierten die Viehzucht sowie der Baumwollanbau mit der damit verbundenen Plantagenwirtschaft. Im amerikanischen Bürgerkrieg (1861-1865) engagierten sich viele prominente Wacoans auf der Seite der Konföderation, weshalb sein Ausgang als bittere Niederlage gegen die Yankees aus dem Norden empfunden wurde. Während sich die Ökonomie nach dem Krieg rasch erholte - unterstützt durch den Bau der Suspension Bridge über den Brazos River und den Anschluss der Stadt an verschiedene Eisenbahnlinien verschärften sich mit dem offiziellen Ende der Sklaverei zusehends die Konflikte zwischen der weißen und der afroamerikanischen Bevölkerung. Immer wieder kam es zu Aufständen und Unruhen. Dennoch wurde die Stadt in der zweiten Hälfte des 19. Jahrhunderts zu einem prosperierenden Zentrum der Agrarproduktion und zu einem wichtigen Verkehrsknotenpunkt und Umschlagsort für Waren und Vieh. 1884 zählte Waco bereits 12.000 Einwohnerinnen; zahlreiche, vorwiegend protestantische Kirchengemeinden formierten sich, und $1886 \mathrm{zog}$ die 1845 in Independence, Texas gegründete Baylor University, die heute die größte baptistische Universität der Welt ist, nach Waco. Mit dem Wachstum der Stadt stieg auch die Kriminalität an, und es entstanden zwielichtige Saloons und Spielhallen sowie ein florierendes Rotlichtviertel. Aufgrund seiner rauen und gewaltsamen Atmosphäre an der räumlich als „frontier“ (Strauss 2017, S. 113) imaginierten europäischen Besiedlungsgrenze erhielt Waco bald den Beinamen „Six Shooter Junction“ (Conger 2016).

Mit Beginn des 20. Jahrhunderts strebte Waco einen Imagewechsel an. Die Handels- und Versicherungswirtschaft wurde gestärkt, und es wurde mehr in Bildung und Wirtschaftsgebäude investiert. 1911 entstand mit dem ALICO Building, einem 22-stöckigen Stahlskelett-Hochhaus für eine Versicherung, das damals höchste 
Abb. 1 Blick in die Webster Avenue in Downtown Waco. Foto: Silke Steets, 2019

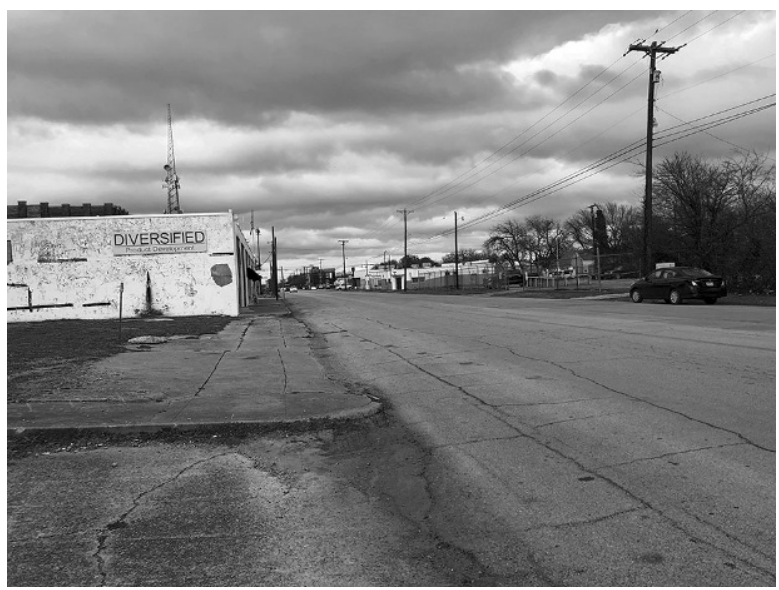

Gebäude in Texas. Die prosperierende Wirtschaft veränderte auch die ethnische $\mathrm{Zu}-$ sammensetzung der Bevölkerung. Immer mehr Afroamerikaner zogen auf der Suche nach besseren Arbeitsplätzen und Bildungsmöglichkeiten vom Land nach Waco, und es bildete sich langsam eine schwarze Mittelschicht. Wohl zum Teil als Reaktion auf diese Entwicklungen wurde Waco in den 1920er-Jahren zu einer zentralen Wirkungsstätte des Ku Klux Klan (Burke 2018). Zwischen 1905 und 1921 kam es in der Stadt zu mehreren Lynchmorden von Weißen an Schwarzen (Carrigan 2004). Tragischer Höhepunkt war die öffentliche Ermordung des 16-jährigen Afroamerikaners Jesse Washington, der am 15. Mai 1916 vor den Augen einer über tausendköpfigen Menge - darunter der Bürgermeister und der Polizeichef der Stadt - brutal verstümmelt, gelyncht und auf einem Scheiterhaufen verbrannt wurde (Bernstein 2005). 1923 zogen mehr als 2000 Mitglieder des Ku Klux Klan in einer Parade durch die Stadt. Die Organisation kontrollierte weite Teile des städtischen Einzelhandels und der Politik. Viele von Wacos Geschäftsleuten und politischen Führern unterstützten den Klan in dieser Zeit zumindest stillschweigend (Burke 2018).

Abb. 2 Waco, Texas - gesehen von der I-35. Foto: Silke Steets, 2019

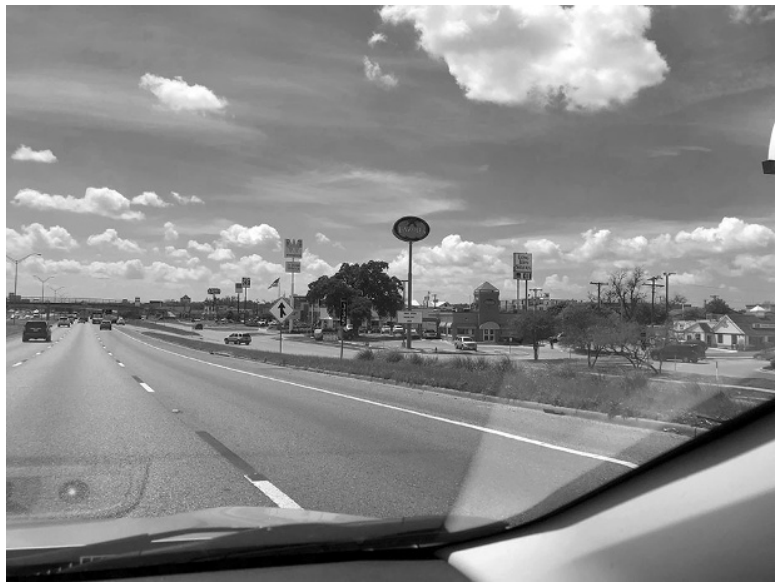


Während die Great Depression die wirtschaftliche Dynamik der Stadt unterbrach und der Zweite Weltkrieg zu ihrer Wiederbelebung beitrug, wurde die jüngere Geschichte Wacos durch zwei Schlüsselereignisse bestimmt: die Verwüstung eines Großteils der Innenstadt durch einen Tornado im Jahr 1953 und die sogenannte „Waco Siege“ 1993. Der Tornado forderte 114 Todesopfer und ließ über Tausend Gebäude zerstört oder beschädigt zurück; die räumlichen Folgen sind noch heute in Form großer ungenutzter Brachflächen und Parkplätze in der wenig verdichteten Innenstadt sichtbar.

Die „Waco Siege“ brachte die Stadt sogar international in die Schlagzeilen. Anlass dafür war die Erstürmung des etwas außerhalb der Stadtgrenze gelegenen Geländes der apokalyptischen Branch-Davidian-Sekte durch das FBI. Knapp 100 Sektenmitglieder, darunter viele Kinder, hatten sich um ihren Anführer David Koresh auf einer Ranch verbarrikadiert und massiv bewaffnet. Das rief die Bundesbehörden auf den Plan. Nach einer über sieben Wochen langen und allabendlich im Fernsehen übertragenen Belagerung stürmte schließlich eine Spezialeinheit das Gelände. Feuer brach aus. Am Ende waren 81 Sektenmitglieder sowie der Sektenführer tot. In der Folge wurde Waco für das liberale Amerika zum Synonym für religiösen Fanatismus. Für das rechte, waffenverrückte Amerika aber standen Waco und David Koresh für den Überlebenskampf gegen die als Besatzungsmacht gedeuteten Bundesbehörden. So wollte etwa der Oklahoma City-Bomber Timothy McVeigh sein Attentat auf das Murrah Federal Building im Jahr 1995 (mit 168 Toten und über 800 Verletzten) explizit als Racheakt für die Geschehnisse von Waco verstanden wissen (Smith et al. 2006).

Sowohl in räumlicher als auch in kulturell-symbolischer Hinsicht ist Downtown Waco, also die eigentliche Mitte der Stadt, seit dem Tornado erstaunlich leer geblieben (vgl. Abb. 1). Stattdessen verdichten sich Restaurants, Cafés und Verkehr rund um dasjenige Infrastrukturprojekt, das Eisenbahnlinien und Flussüberquerungen Mitte der 1950er-Jahre als Wirtschaftsmotor abgelöst hat: die Interstate-Autobahn. Waco liegt an der I-35, die die USA einmal komplett in Nord-Süd-Richtung durchquert - von Duluth, Minnesota an der Grenze zu Kanada bis Laredo, Texas an der Grenze zu Mexiko. Als Verbindung zwischen Dallas/Fort Worth im Norden und

Abb. 3 Waco, Texas - beworben an der I-35. Foto: Silke Steets, 2019

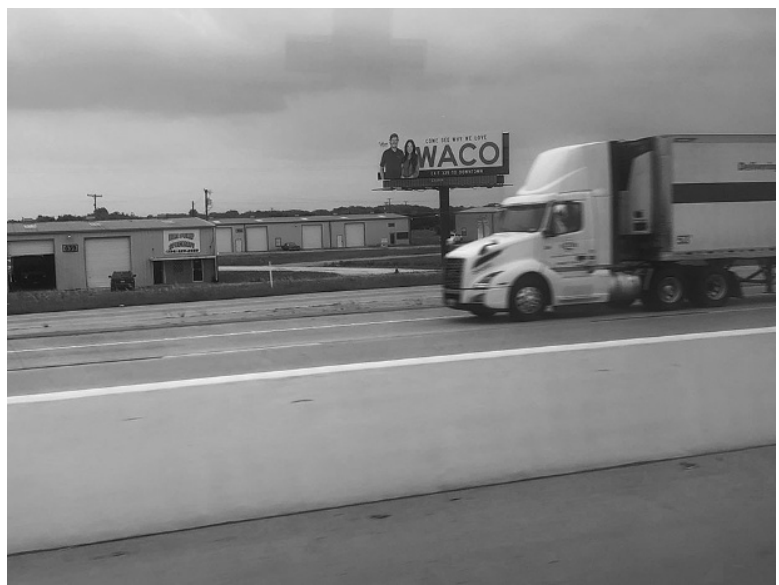


Abb. 4 Chip und Joanna Gaines: Aktuelle Buchcover. Quelle: https://shop.magnolia. com/collections/magnoliapublications, zuletzt aufgerufen: 25. April 2021
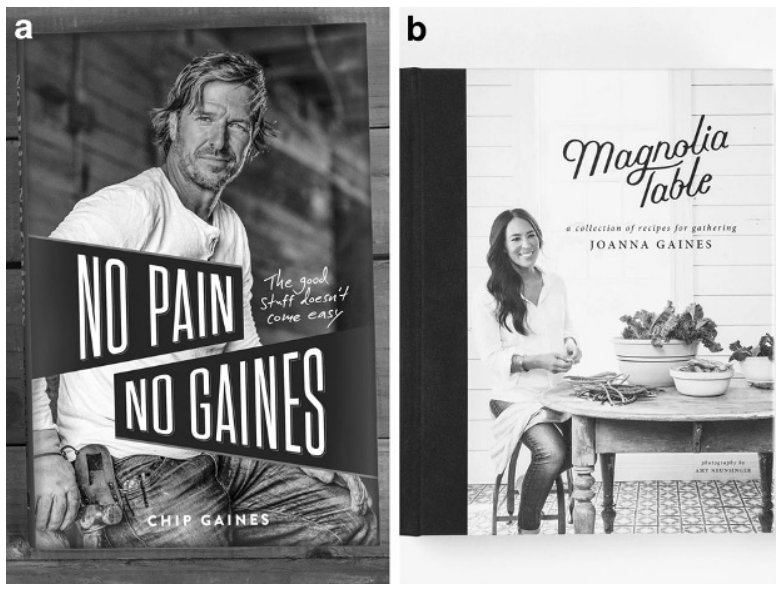

Austin/San Antonio im Süden ist sie zugleich eine der wichtigsten Verkehrsadern Texas.

Im Bereich um Waco passieren die Trasse etwa 140.000 Fahrzeuge täglich (Ray 2019) - und damit mindestens ebenso viele potenzielle Abnehmerinnen für Werbebotschaften aller Art sowie Rastsuchende auf der Durchreise. Die Raumlogik der Autobahn entspricht dem Modell des ,commercial strip“ (Jakle 2010, S. 418): Niedrige, großflächige Gebäude, dominiert von riesigen Schildern, winken Autofahrer auf die angrenzenden Parkplätze (vgl. Abb. 2). Während dieser die Stadt in Nord-SüdRichtung durchschneidende „Bahnenraum“ (Löw 2020) ganz auf die automobilisierte Wahrnehmung (Venturi et al. 2007) und die Bedürfnisse der Durchreisenden ausgerichtet ist, macht er aus Waco selbst vor allem eins: einen „Nicht-Ort“ (Augé 1994), also einen Ort ohne benennbare Qualitäten, den man tausendfach repliziert an amerikanischen Autobahnen findet und der nichts weiter ist als ein beliebiger „,stopover on the way up and down I-35“ (Petersen 2019).

\section{Chip und Joanna Gaines und das Fixer Upper-Narrativ}

Dies ändert sich jedoch seit einigen Jahren. Nähert man sich Waco von Norden über die I-35, grüßt einen bereits rund zehn Meilen vor der Stadtgrenze ein junges sympathisches Paar von einer Werbetafel mit den Worten: „Come see why we love Waco“ (vgl. Abb. 3).

Bei dem Paar handelt es sich um Chip und Joanna Gaines, die Waco seit 2013 mit ihrer TV-Show Fixer Upper auf völlig neue Art national wie international bekannt gemacht haben. Fixer Upper ist eine Hausrenovierungs- und Innenarchitekturshow, in der das Paar heruntergekommene Häuser in und um Waco aufspürt und für ausgesuchte Kundinnen entrümpelt, umgestaltet, saniert und einrichtet, um die so erneuerten Häuser schließlich an ihre stets überwältigten neuen Eigentümer zu übergeben. Die Show, die von 2013 bis 2018 in fünf Staffeln mit insgesamt 79 Folgen auf dem Kabelkanal HGTV ausgestrahlt wurde, hat in den USA bis zu 75 Mio. Zuschaue- 
Abb. 5 Weiß gestrichene Shiplaps und Metallschilder - Szene aus dem Fixer Upper-Merchandising Store in Waco. Foto: Silke Steets, 2019

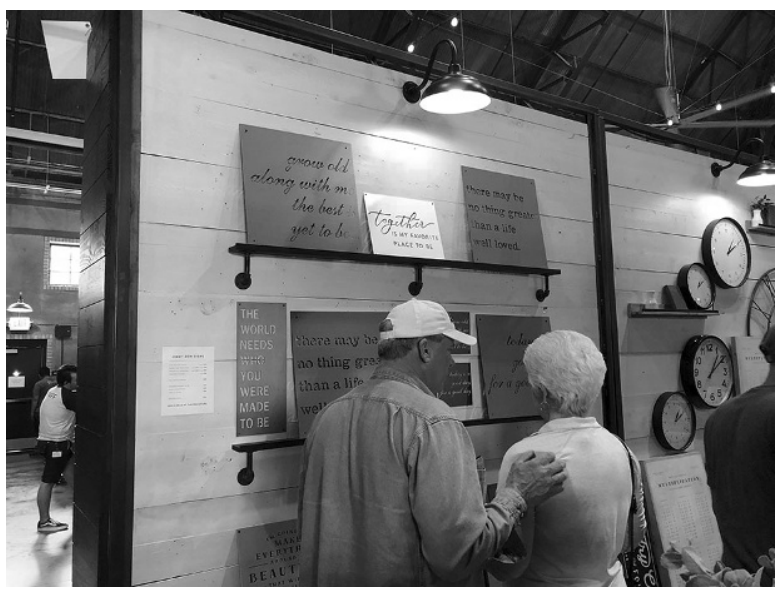

rinnen pro Staffel gefunden (Magnolia Network 2020); im deutschen Fernsehen läuft sie auf dem Spartensender Sixx. Um Reichweite und Popularität der Show zu erklären, wird meist die gegenwärtige Trendigkeit eines ästhetisierten Landlebens und des damit verknüpften „Farmhouse Chic“ verwiesen, doch scheinen bei näherer Betrachtung auch die spezifische (geschlechtliche) Dynamik zwischen den Hauptfiguren Chip und Joanna und vor allem die Erzählstruktur von Fixer Upper von Bedeutung zu sein. Bevor die räumlichen Auswirkungen des Fixer Upper-Booms auf Waco beschrieben werden, gilt es daher zunächst, einen genaueren Blick auf die Show zu richten.

\subsection{Genderrollen und Paardynamiken}

Die Dynamik zwischen Chip und Joanna Gaines ist schnell erzählt: Chip ist gleichzeitig großer Junge und starker Mann. Er albert gerne herum, nimmt das Leben leicht, geht Risiken ein und ist sich für keinen $\mathrm{Spa} 3 \mathrm{zu}$ schade. Gleichzeitig ist er ein kompetenter und hart arbeitender Handwerker (vgl. Abb. 4), er weiß, wie man Pläne in die Tat umsetzt, und verkörpert den Prototyp des modernen Cowboys. Als solcher sitzt er freilich nicht mehr auf dem Rücken eines Pferdes, sondern fährt einen möglichst großen (Texas Edition!) Pick-Up Truck, auf dessen Ladefläche er Material und Maschinen transportiert, die er braucht, wenn er mit der eigenen Hände Arbeit etwas erschaffen will - oder etwas abreißen darf: Mit dem „Demo-Day“, dem „Demolition Day“, dem Abrisstag als der Inszenierung archaischer Männlichkeit, beginnt jede Fixer Upper-Hausrenovierung.

Joanna hingegen ist die moderne, feminine, äußerst design-kompetente, stets Kosten und Risiken kalkulierende, aber dennoch humorvolle Frau an Chips Seite (vgl. Abb. 4). Sie ist es, die die Gestaltungshoheit über die Umbauprojekte hat und weiß, wie sie ihre Vorstellungen (von Chip) umgesetzt bekommt. Wenn er mal wieder herumalbert, fragt sie ihn in aller Ruhe, ob die elektrische Verkabelung rechtzeitig eintrifft, damit man das riesige Wagenrad aufhängen kann, das als Kronleuchter vorgesehen ist. Ihre gestalterische Handschrift im modernen Landhausstil trägt als 
wichtigstes Kennzeichen weiß gestrichene Shiplaps (Vertäfelungen aus aufbereitetem Altholz), ansonsten sichtbare Ziegel und Balken, offene Grundrisse, Kücheninseln und Schilder aus Metall oder Holz, die entweder einen tieferen Lebenssinn zum Ausdruck bringen oder den Zweck eines Raumes oder Raumelements ankündigen (vgl. Abb. 5).

Zusammen sind Chip und Joanna nicht nur das sympathische Ehepaar aus Fixer Upper, sondern - wie man schnell über die von ihnen vielfältig bespielten sozialen Medien herausfindet - auch Absolventen der Baylor University, wiedergeborene Christen, Mitglieder der evangelikalen Antioch Community Church von Waco, Inhaber des mittlerweile millionenschweren und weitverzweigten Unternehmens $M a$ gnolia Inc. und Eltern von fünf Kindern im Alter zwischen zwei und 17 Jahren. Die Familie wohnt auf einer selbst renovierten Farm am Rande der Stadt und verkörpert das Ideal einer ebenso modernen wie konservativen und dabei ökonomisch überaus erfolgreichen texanischen Familie mit US-weitem Celebrity Status. ${ }^{3}$

\subsection{Die Struktur des Fixer Upper-Narrativs}

Die einzelnen Folgen von Fixer Upper sind entlang einer idealtypischen Erzählstruktur aufgebaut, deren Eckpunkte ich zur besseren Nachvollziehbarkeit im Folgenden kursiv setze: Jede Folge beginnt mit der Vorstellung eines Paares, das sich ein individuell gestaltetes Zuhause wünscht und ein eigenes Budget mitbringt, um Hauskauf und Umbau zu finanzieren. ${ }^{4}$ Daraufhin suchen Chip und Joanna drei geeignete, aber unterschiedliche Objekte aus, die zu viert besichtigt werden. Während der Begehung der mal mehr, mal weniger heruntergekommenen Häuser ist es Joannas Aufgabe, das wenngleich oft schwer fassbare Potenzial eines Objekts herauszustellen, sodass sich Zuschauerinnen wie Hauskäufer vorstellen können, wie ein Haus nach seiner Restauration erstrahlen könnte. Ist die Entscheidung für ein Objekt gefallen, schauen sich Chip und Joanna das Haus genauer an. Verschiedentlich tauchen Probleme auf (ein verdeckter Wasserschaden hier, ein Bienenvolk in der Fassadenverkleidung

\footnotetext{
${ }^{3}$ Die Verbindung von „,modern“ und „konservativ“ ließe sich freilich noch genauer herausarbeiten. Aus Platzgründen nenne ich hier exemplarisch die wiederkehrende Betonung von Joannas Doppelrolle als eigenständiger Businessfrau und traditionsbewusster Hausfrau und Mutter: „Between running Magnolia and her many projects, Joanna enjoys life on the farm, cooking family recipes in her kitchen, and gardening. Above all, she loves being with her five kids"(vgl. https://magnolia.com/about/, zuletzt aufgerufen: 13 . Februar 2021). Über ihren Erfolg und das wachsende Berühmtsein reflektierten Chip und Joanna Gaines publikumswirksam im März 2021 in der TV-Show Super Soul von Talk-Ikone Oprah Winfrey.

4 Über die Auswahl der Kandidatinnen für Fixer Upper gibt es teils hitzige Debatten in den sozialen Medien. So wurde immer wieder die Vermutung geäußert, dass man (wie die Gaines auch) Mitglied der Antioch Community Church von Waco sein müsse (zu dieser Kirche unten mehr), aus deren Kreis tatsächlich viele Hauseigentümerinnen stammen. Auffällig ist, dass ausschließlich heterosexuelle und überwiegend weiße Paare zu sehen sind. In den Trailern der neuen, ab Juli 2021 von Magnolia Network (auch dazu unten mehr) ausgestrahlten Shows rund um Heim, Essen, Garten und Renovierung scheinen jedoch auch vielfältigere Lebensentwürfe repräsentiert zu sein (vgl. https://p.magnolia.com/network/\#magnolia-network-originals, zuletzt aufgerufen: 14. Februar 2021). Das Mindestbudget für Bewerberinnen der im Herbst 2021 startenden neuen Fixer Upper-Staffel beträgt: 50.000 Dollar für die Renovierung plus die Kosten für den Erwerb einer geeigneten Immobilie (https://p.magnolia.com/network/\#frequently-asked-questions, zuletzt aufgerufen: 14. Februar 2021). Die hier wie im Abschnitt zur Stadtgeschichte bereits angedeutete Hegemonie des weißen evangelikalen Milieus in Waco wird unten weiter ausgeführt.
} 
dort etc.), die technisch (Chip) und/oder gestalterisch (Joanna) gelöst werden. Dann folgt der bereits angedeutete „Demo-Day“, der Abrisstag, der von Chip und seinem Team durchgeführt wird und der an ein Reinigungsritual (Douglas 1985) erinnert: Verkleidungen, Fußbodenbeläge, überflüssige Einbauten und ganze Wände werden genüsslich-schwitzend herausgerissen, bis das Potenzial des Hauses freigelegt ist. Indem Schmutz entfernt, Wertvolles von Wertlosem und Reines von Unreinem getrennt wird, entfaltet sich sukzessive eine sowohl räumliche wie symbolische Neuordnung des Hauses (ebd.). Währenddessen erarbeitet Joanna am Computer eine visualisierte Darstellung des fertig umgebauten Hauses und stimmt sie mit den Hauseigentümern ab. Sind die Details geklärt, beginnt der eigentliche Umbau, den wiederum Chip und seine Männer durchführen. Auf ihren regelmäßigen Stippvisiten findet Joanna auf der Baustelle meist etwas zu kritisieren, und es setzt das für die Show so charakteristische flirtend-neckende Hin und Her zwischen Chip und Joanna ein, an dessen Ende immer eine pfiffige Lösung für das identifizierte Problem steht. Noch während der Umbauphase macht sich Joanna auf die Suche nach passenden und auf die Persönlichkeit der Hauseigentümer abgestimmten Einrichtungsgegenständen. Dafür durchforstet sie die Antiquitätenshops in und rund um Waco und arbeitet mit ausgesuchten lokalen Handwerksbetrieben zusammen. ${ }^{5}$ Den letzten Abend vor der Übergabe verbringt Joanna in einer Art spirituellem Abschlussritual alleine im Haus. Die mit viel Dreck und Kraftaufwand verbundene räumlich-symbolische Neuordnung endet in einem Moment der Ruhe und Reflexion, in dem Joanna allerletzte Details richtet, um - ähnlich dem Verpacken eines Geschenkes - dem perfekt auf die Persönlichkeit der Eigentümerinnen abgestimmten Objekt den letzten Schliff zu verpassen. Gleichzeitig deutet Joanna an, wie die neugestalteten Räume wohnend genutzt werden können. Rituell bewegt sich dieser letzte Abend auf der Schwelle zwischen Neuordnung (Abschluss eines Hausumbaus) und imaginierter Aneignung dieser Neuordnung (Bewohnen). Interessanterweise wird diese Schwellensituation (Van Gennep 1986) im rituellen Modus des Schenkens (Berking 1996) dargestellt, denn es sind nicht Chip und Joanna, die das umgebaute Haus beziehen, sondern die neuen Eigentümer, zu denen es perfekt passen soll. Allerdings bedeutet Schenken, wie Helmuth Berking (ebd.: 10) betont, immer auch ,eine Macht erwerben, einen symbolischen Tausch realisieren, Bindungen und Bündnisse initiieren, Rechte und Pflichten attribuieren, subjektive Bedeutungen objektivieren und Alter ego systematisch klassifizieren“ (ebd.: 10). Die eigentliche Übergabe ist als Vorher-NachherShow inszeniert: Vor dem Haus wird eine große zweiteilige Leinwand mit dem Vorher-Zustand auf fahrbaren Unterbauten errichtet, die sich den gespannten Hausbesitzern wie ein Vorhang öffnet. Sowohl der Anblick von außen auf das Fixer Upper als auch die gemeinsame Besichtigung des Inneren lässt durch die Bank begeisterte

\footnotetext{
5 Eine Folge der Popularität von Fixer Upper ist, dass bestimmte von Joanna in der Show aufgesuchte Antiquitätenläden in und um Waco nur noch an drei Tagen im Monat öffnen, weil sie allein in dieser Zeit von Fixer Upper-Fans komplett leer gekauft werden. Der Schreiner Clint Harp sowie der Metallschlosser Jimmy Don, die beide regelmäßig von Joanna für Spezialanfertigungen beauftragt werden, sind zu nationalen Berühmtheiten geworden. Harp schreibt Bücher und geht damit auf Tournee durch die USA, Don tritt regelmäßig als Figur (und Selfie-Motiv) ,,zufällig“ bei den Waco-Tours-Stadtrundfahrten auf (dazu unten mehr).
} 
(„Oh my Gosh! - This is amazing!“) und nicht selten zu Tränen gerührte Beschenkte zurück.

Fasst man die Erzählstruktur von Fixer Upper etwas abstrakter, lässt sie sich darstellen als Sequenz folgender Schritte: (1) Inspektion eines verwahrlosten, aber mit Potenzial ausgestatteten Ausgangsobjekts, (2) Entscheidung, das Objekt trotz zu erwartender Kosten, Probleme und Mühen zu restaurieren, (3) rituelle Reinigung des Objekts am Demo-Day, (4) Entwicklung einer Vision für eine bessere Version des Objekts, (5) mühsamer Umbau mit neu auftauchenden, aber lösbaren Problemen, (6) ritueller Abschluss des Umbaus im Modus des Schenkens und schließlich (7) Überwältigung beim Anblick des erfolgreich erneuerten Hauses im Moment des Beschenktwerdens. Religionssoziologisch betrachtet fällt die Ähnlichkeit dieser Erzählstruktur mit der Struktur evangelikaler Konversionserzählungen ins Auge. Bernd Ulmer (1998), der Konversionserzählungen im Hinblick auf ihre Gattungsmerkmale untersucht hat, weist allgemein auf eine dreigliedrige Zeitstruktur hin, bei der ein „Wendepunkt“ die Erzählung in eine „Zeit davor“ und in eine „Zeit danach“ aufteilt. Inhaltlich ist die erzählte „Zeit davor“ durch die Thematisierung von Krisenerfahrungen und Aussichtslosigkeit geprägt und im Rückblick negativ besetzt. Der „Wendepunkt“, also die eigentliche Bekehrung, wird als überwältigendes, schwer zu verbalisierendes und hochemotionales Erlebnis beschrieben; die „Zeit danach“ ist erzählstrukturell durch die Darstellung der heilenden Wirkung der Krisenlösung und durch die Dominanz der neuentwickelten (religiösen) Weltsicht geprägt. Im Fall des Fixer Upper-Narrativs kommen noch einige inhaltliche Elemente hinzu, die man analog auch in individuellen Konversionserzählungen zum evangelikalen Christentum findet (Steets 2020). Dazu zählen die Vorstellung, dass jede Person, egal, wie sündig sie auch lebt (bzw. jedes Haus, egal, wie heruntergekommen es auch ist), ein Potenzial aufweist, das es sich lohnt zu heben, oder die Überzeugung, dass vor jedem Wendepunkt die bewusste und Verantwortung übernehmende Entscheidung für einen Neuanfang steht, oder aber das Bewusstsein darüber, dass der Erneuerungsprozess (,restoration“) in der Regel mit auftauchenden Problemen verbunden ist, die es sich aber ebenfalls lohnt zu bearbeiten, um schließlich mit einer krisenbeendenden und heilenden Wiedergeburt belohnt zu werden, die als Geschenk Gottes erlebt wird (Kiefer 2020). Während in Fixer Upper die Phase nach dem Wendepunkt, also das Leben im erneuerten Haus, allenfalls angedeutet wird, liegt der Schwerpunkt der filmischen Inszenierung auf der sich vorwiegend im Verborgenen vollziehenden Arbeit an der Erneuerung, wobei alles auf die - dann wiederum stolz nach außen kommunizierte - Darstellung der gelungenen Umwandlung des Hauses als dem emotionalen Höhepunkt einer jeden Folge hinausläuft. Seine religiöse Konnotation erhält Fixer Upper zum einen durch das Zusammenspiel dieser Elemente, wodurch eine spezifisch evangelikale Erzählstruktur der Erneuerung entsteht, die das vordergründig verhandelte Thema der Hausrenovierung grundiert, und zum anderen durch die rituelle Herstellung und massenmediale Darstellung einer räumlichsymbolischen Neuordnung des Hauses, die das „Chaos“ des baulichen Niedergangs korrigiert (Douglas 1985).

Eine solche implizite Kommunikationsform des Religiösen, die sich erstens weit jenseits des klassischen Feldes der Religion vollzieht und zweitens Elemente der Popkultur (TV-Show) mit religiösen Motiven (Erneuerung, Heilung) verbindet, lässt 
sich als zeitgenössische Ausprägung der „Populären Religion“ (Knoblauch 2009) deuten. Der Begriff zielt darauf ab, religiöse Erfahrungen und Praktiken, die aufgrund ihrer popkulturellen Codierung nicht mehr unmittelbar als solche erkennbar sind, als neue Sozialform des Religiösen (Luckmann 1991) sichtbar zu machen. Wie sich noch zeigen wird, entsteht die im vorliegenden Fall untersuchte neue Sozialform der Populären Religion im Schnittfeld von Evangelikalismus, Medien, Tourismus, Sentimentalität und (materiellem) Raum.

Während Fixer Upper auf religiöse Motive von Erneuerung und Heilung anspielt, die für nichtreligiöse Fans vermutlich in der Tat implizit bleiben, weil sie weder das Narrativ noch die rituelle Neuordnung so ohne weiteres als evangelikal entschlüsseln können, werden diese Motive in evangelikalen Milieus durchaus verstanden und sogar explizit aufgegriffen. So postete die Open Door Church aus Chillicothe, Ohio auf ihrer Facebook-Seite ein Predigtvideo mit dem Titel ,Life is the Ultimate Fixer Upper"6, die Northgate Church aus Pittsburgh, Pennsylvania spricht in ihrem Facebook-Auftritt von einem „Fixer Upper with God“"7, und die Liquid Church aus New Jersey hat auf ihrem Youtube-Kanal unter dem Titel „Fixer Upper - Rebuild your world in 52 days" gar eine eigene Predigtreihe aufgelegt ${ }^{8}$, in der Parallelen zwischen der Show und dem Wiederaufbau Jerusalems (beschrieben in der Bibel im Buch Nehemia) gezogen werden. Noch deutlicher wird ein Blog-Beitrag auf der christlichen Plattform bible.org:

I love [Fixer Upper, Anm. Steets] because Chip and Joanna revitalize dilapidated houses into beautiful homes. I also love it because the show reminds me of what God has done in the life of a believer. [...] Next time you watch the show, remember God took our deteriorating, dying souls and made them new - He made us beautiful! Every single moment of our lives God works in us and lavishes us with His love. And He adorns us with courage, compassion and strength (and so much more) so He can display to the rest of the world His handiwork - so we can go out and show the world our worth because of what God has done in our lives (Wroten 2015).

Die Beispiele zeigen, dass der Erfolg von Fixer Upper in der Lage ist, konservative Christen in den USA regelrecht zu elektrisieren, und dass die Show zu einer - teils expliziten, teils impliziten - Popularisierung des evangelikalen Erneuerungsmotivs beigetragen hat. Darüber hinaus hat sie die erstaunliche ,Wiedergeburt“ der Stadt Waco eingeleitet.

\section{Die Politik der coolen evangelikalen Urbanität}

Verlässt man den Bahnenraum der Interstate 35 und besucht Waco, dann findet man sich in einer Stadt wieder, deren Imaginaire sich am Modell der amerikanischen

\footnotetext{
6 https://www.facebook.com/watch/live/?v=302596917004178\&ref=watch_permalink, zuletzt aufgerufen: 23. April 2021.

7 https://www.facebook.com/watch/?v=975584386292011, zuletzt aufgerufen: 23. April 2021.

8 https://www.youtube.com/watch?v=0he1WCzARwM, zuletzt aufgerufen: 23. April 2021.
} 
Small Town orientiert. Das heißt, sie lässt sich situieren ,somewhere between the countryside and the metropolis“ (Strauss 2017, S. 190) und wird von Menschen bewohnt, ,who have rural traditions and urban aspirations“ (ebd.). Allerdings wandelt sich Waco rasant. Dieser Wandel erfasst inzwischen auch Milieus, die mit dem evangelikalen Erneuerungsnarrativ wenig anzufangen wissen. Kate*, Anfang 50, violett gefärbte Haare, buntes T-Shirt mit „I love Texas“-Aufschrift, das Auto übersät mit feministischen und antirassistischen bumper stickers, ist Mitglied der liberalen unitarischen Gemeinde Wacos. ${ }^{9}$ In den 1980er-Jahren hat sie in Austin gelebt, der texanischen Hauptstadt, die um die Jahrtausendwende aufgrund ihrer vielfältigen Musik- und Kreativszene zu einem der beliebtesten Orte der Creative Class (Florida 2002) in den USA wurde. Im Interview zieht Kate* Parallelen zwischen Austin in den 1980er-Jahren und Waco heute:

Austin had that sort of funky vibe. You know, back in the 80s, you know, cause [...] they had been an oil town and then the oil went bust in the 80s and so Austin was just this weird, funky art town that also happened to have the capital in it. And the people in the capital are very conservative. But [...] the rest of Austin is very liberal. But it was also kind of like (.) there was a lot of poverty, Austin still has a lot of issues of not acknowledging the racial issues they have. (.) But you know, then they kind of had their technology boom and it's been amazing and terrible, (laughs) both at the same time. But Waco to me right now feels like Austin felt in the early 80s. Like there's starting to be an art scene and a music scene and there's like different events coming up and there's different groups gathering. [...] You know the fact that I've gotten two invitations to

Abb. 6 Magnolia Market at the Silos. Foto: Silke Steets, 2019

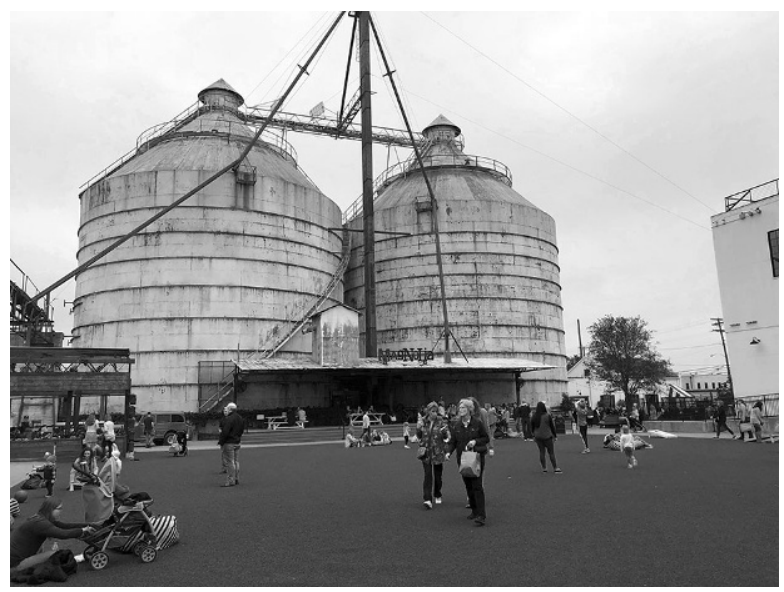

\footnotetext{
9 Ich treffe Kate* im Mai 2019 zu einem Interview über liberale religiöse Strömungen in Waco. Wir verabreden uns in einem Café in Downtown, wo sie im beschriebenen Outfit erscheint. Kate* gehört der Unitarian Universalist Fellowship von Waco an. Der Unitarian Universalism gilt als äußerst liberale Form der Religion. Ursprünglich eine protestantische Abspaltung, teilen Unitarierinnen kein gemeinsames Glaubensbekenntnis (mehr) und kennen keine heiligen Schriften, vielmehr einigt sie das Streben nach spiritueller Weiterentwicklung und nach einem liebe- und respektvollen Umgang miteinander. Unter ihnen findet man Agnostiker ebenso wie Gläubige und Atheistinnen.
} 
Abb. 7 The Shot Gun House, buchbar über die Plattform Airbnb. Foto: Silke Steets, 2019

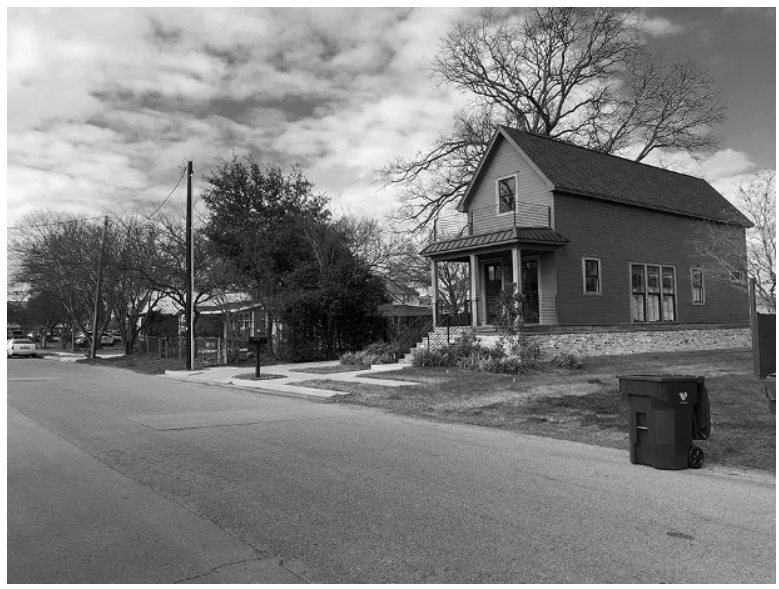

drum circles this month, it's like real good (laughs). You know? (Int_Kate*: Zeilen 765-775).

Indem sie das Austin der 1980er-Jahre mit dem Waco von heute vergleicht, zeichnet Kate* das Bild einer Stadt, in der sich gerade jener kreative „Humus“ herausbildet, der für die Entwicklung einer Creative City (Landry 2000), also einer Stadt, in der die Kultur- und Kreativwirtschaft das ökonomische Wachstum antreibt, als unverzichtbar gilt. Stadträumlicher Motor dieser Entwicklung in Waco ist zweifelsohne Magnolia Market at the Silos, ein Downtown Revitalisierungsprojekt, das Chip und Joanna Gaines 2015 im Zug ihres Fixer Upper-Erfolges realisiert haben. Auf dem mehrere Blocks umfassenden Gelände zweier ehemaliger Baumwollsamendepots, den Silos, erstreckt sich heute eine postindustrielle Shoppingund Freizeitlandschaft, die an die Cool Urbanity-Leitbilder der 2000er-Jahre (Steets 2005) und die Ästhetisierung öffentlicher Räume für die New Urban Middle Class in Metropolen wie New York City erinnert (Zukin 1995). Im Fall von Waco spricht man inzwischen überregional vom „Magnolia Effect“ (Sachs 2020).

\subsection{Der Magnolia Effekt}

Das Gelände von Magnolia Market at the Silos (vgl. Abb. 6) umfasst ein großes Ladengeschäft für Einrichtungsgegenstände und Fixer Upper-Merchandising, die Bäckerei Silos Baking Co. sowie einen Außenbereich mit Garten, Food Trucks, Picknick-Tischen und einer Kunstrasenspielfläche für große und kleine Kinder. 2019 zählte Magnolia Market geschätzte 40.000 Besucherinnen in der Woche, Tendenz (vor Corona) steigend. Mittlerweile beschäftigt das Gaines'sche Unternehmen Magnolia Inc. allein in Waco über 750 Menschen (Petersen 2019). Neben Magnolia Market setzt es sich aus der Immobilienfirma Magnolia Realty (seit 2009), dem Online-Shop Magnolia.com (seit 2014), dem Bed and Breakfast Magnolia House (seit 2016), dem Restaurant Magnolia Table (seit 2018), dem Little Shop on Bosque (Joannas erstem Ladengeschäft in North Waco, wiedereröffnet 2018), mehreren zur Vermietung angebotenen Ferienhäusern (seit 2017) und dem Café Magnolia Press 
(seit 2019) zusammen. Im Aufbau befinden sich das Boutique Hotel Magnolia Hotel (Eröffnung 2021) sowie eine Medienabteilung, die bereits das vierteljährlich erscheinende Magnolia Journal (seit 2016, Auflage 5,6 Mio. Exemplare) sowie Chips und Joannas Bücher (bislang acht, siehe Abb. 4) produziert und vertreibt, dazu diverse Kanäle auf Twitter, Facebook und Instagram füttert und den TV-Kabelsender $M a$ gnolia Network vorbereitet. Dieser wird von Mitte 2021 an eine Neuauflage von Fixer Upper sowie weitere Shows zu Themen wie Beziehung, Familie, Elternschaft, Garten, Kochen und Inneneinrichtung produzieren. Der geschätzte Marktwert von Chip und Joanna Gaines liegt aktuell bei rund 20 Mio. US-Dollar (Barth 2020).

In Folge dieses Booms haben sich nicht nur viele weitere kleine Geschäfte für Antiquitäten, Mode, Essen und Lebensstil gegründet, es sind auch einige Kunstgalerien, Fahrradläden, Co-Working Spaces (und offenbar auch Drum Circles) entstanden. Vor allem aber hat sich die Zahl der Touristinnen in den letzten sechs Jahren vervielfacht. 2018 besuchten insgesamt rund 2,6 Mio. Menschen die Stadt, das sind im Wochendurchschnitt etwa 50.000, bei jährlichen Steigerungsraten im zweistelligen Bereich (Petersen 2019). Während Waco 2018 die höchste Hotelauslastungsquote in ganz Texas hatte (Heft 2019), übernachtet das Gros der Touristen - dem authentischen Fixer Upper Feeling auf der Spur - allerdings vorzugsweise in liebevoll renovierten Holzhäusern. Dies wiederum ließ die Zahl der privat vermieteten Ferienwohnungen in die Höhe schnellen. 2018 erweiterte sich das Angebot auf der Plattform Airbnb um etwa ein Haus pro Woche, Schätzungen zufolge gab es dort 2019 rund 450 private Häuser und Wohnungen für temporäres Wohnen, die rund 3,6 Mio. US-Dollar in die Taschen der Wacoans spülten (Heft 2019). Während man noch 2015 allenfalls eine Handvoll privater Übernachtungsmöglichkeiten in Waco fand, sind heute auf Airbnb sogar einige begehrte Fixer Upper-Originale buchbar, darunter The Shot Gun House (vgl. Abb. 7), The Giraffe House oder The German Schmear Cottage.

Die meisten (anderen) Unterkünfte werben bereits im Titel mit ihrer Nähe zu $M a$ gnolia Market oder mit besonderen Designmerkmalen (z. B. Tiny Houses). Auffällig ist die hohe Quote an „Superhosts“, also an besonders erfahrenen Gastgebern, die meist mehrere Objekte professionell-gewerblich vermieten und überdurchschnittlich viele herausragende Bewertungen erhalten. In ihren Airbnb-Profilen wird die traditionell leidenschaftliche texanische Gastfreundschaft (Wright 2018) nicht selten mit der eigenen religiösen Orientierung und fast immer mit dem Stolz, in Waco zu Hause zu sein, begründet. Letzteres wäre noch vor zehn Jahren, als Waco vor allem mit der ,Waco Siege“ assoziiert wurde, undenkbar gewesen. ${ }^{10}$

\footnotetext{
10 Als Beispiel mag die Selbstdarstellung von Jeff und Sara Jones als Airbnb-Gastgeber dienen. Sie vermieten zwei Objekte, darunter ein Fixer Upper-Original, sind Mitglieder der Antioch Community Church und schreiben über sich selbst: „Hi, we are Jeff and Sara Jones. Baylor University and our church here in Waco allowed us to cross paths. Not too long after college we both made the trek to Knoxville, Tennessee. As college ministry staff with a local church, we had the privilege to work with some incredible students for two years. After much transition, we are now back in Waco, Texas and proud to call it home. This city is quaint, friendly, and ever growing." (vgl. https://www.airbnb.de/rooms/24629664?source_impression_ id=p3_1616505794_\%2BPF8mYhh8F5k9HDq\&guests=1\&adults=1, Zuletzt aufgerufen: 23. März 2021). Die Darstellung der eigenen Biographie in Metaphern der Wegkreuzung, der Transition und des glücklichen Ankommens spielt ebenfalls Elemente evangelikaler Konversionserzählungen an.
} 
Abb. 8 Die Gründerinnen von Waco Tours, wie sie sich auf ihrer Website präsentieren (von links nach rechts): Rachel und Luke Whyte sowie Rachel und David Ridley. Quelle: https://www.waco-tours.com/ourstory, zuletzt zugegriffen: 29. Mai 2021

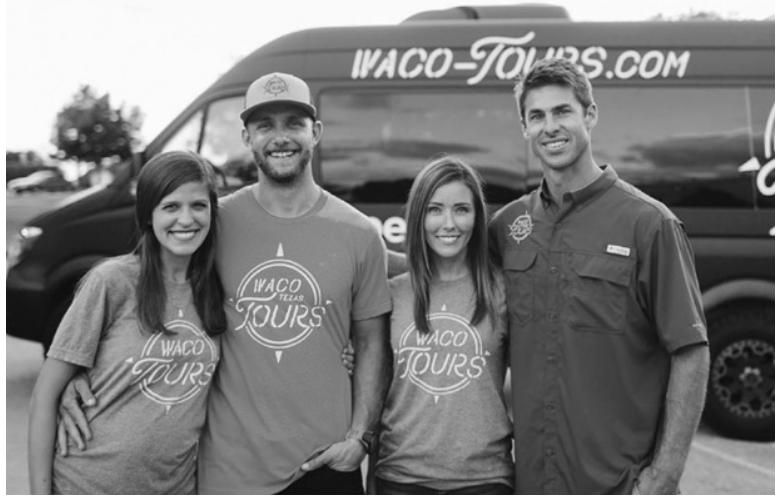

Eine zunehmend kritisch diskutierte Folge des „Magnolia Effekts“ und der hohen Nachfrage nach Häusern und Wohnungen für touristische Nutzungen ist die rasante Entwicklung der Marktpreise - sowohl für Immobilien als auch für Grundstücke. Zwar ist der Wohnungsmarkt in Waco kein klassischer Mietmarkt wie in Deutschland, und viele Menschen besitzen die Häuser, in denen sie leben; allerdings führen die an den Wert einer Immobilie gekoppelten und eben auch spürbar steigenden property taxes bereits zur Verdrängung ärmerer Bevölkerungsgruppen (Lawson 2020). Unter Druck gerät derzeit insbesondere der Stadtteil East Waco, der nordöstlich des Brazos River direkt an Downtown und die Baylor University angrenzt und historisch und kulturell vorwiegend afroamerikanisch geprägt ist. Von dieser Seite des Flusses aus betrachtet, der in Waco auch die Color Line bildet, verursacht der raumgreifende Magnolia-Boom zunehmend Angst vor Verdrängung und vor der Zerstörung der über Jahrzehnte relativ unbeachtet und ungestört sich entwickelnden schwarzen Lokalkultur (The Waco History Podcast 2019). Hier deutet sich bereits etwas an, was sich im Folgenden noch erhärten wird: Die „Wiedergeburt“"Wacos ist vor allem eine von Weißen erzählte Geschichte.

\subsection{Waco Reborn oder: Bist Du das, Gott?}

Ausgesprochen enthusiastisch wird diese Geschichte bei den Stadtführungen von Waco Tours erzählt. Gegründet wurde Waco Tours 2016 von zwei befreundeten Ehepaaren: Luke und Rachel Whyte sowie David und Rachel Ridley (vgl. Abb. 8), die - alle weiß, sportlich, mit College-Abschluss und in ihren Dreißigern - sich im Studium an der Baylor University oder in der Antioch Community Church kennengelernt haben. Das Unternehmen beschäftigt mittlerweile rund siebzig Mitarbeitende und hat in den fünf Jahren seines Bestehens mehr als 55.000 Gäste ,,all the hidden gems of Waco“ gezeigt (Waco Tours 2021). Im persönlichen Umgang mit seinen Gästen 
Abb. 9 North Waco als Ort noch zu hebender Potenziale: Verfallendes Haus (a), Schild: „I Pay Top Dollar 4 Houses“ mit Telefonnummer aus Dallas (b), Fotos: Silke Steets, 2019
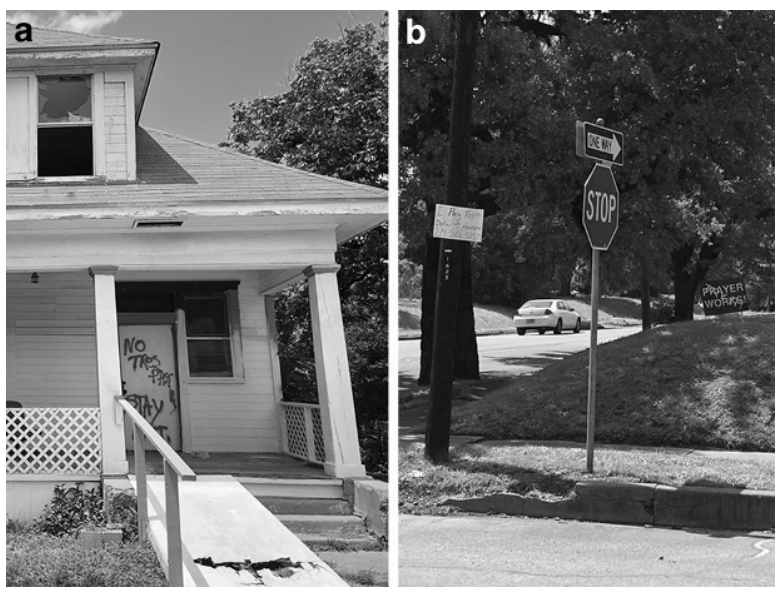

legt das Unternehmen großen Wert auf eine herzliche, persönliche und familiäre Atmosphäre, was offenbar gut ankommt. ${ }^{11}$

Auch ich nehme, einmal im Februar und einmal im Mai 2019, an der rund zweieinhalbstündigen Classic Tour für 79 US-Dollar teil - und finde mich wieder als einzige Alleinreisende unter (wieder)verliebten Pärchen, Freundinnengruppen und Kleinfamilien. Meine Mitreisenden, allesamt Weiße, kommen aus Utah, Colorado, Florida, Oklahoma und sogar aus Kanada. Die Tour beginnt mit einer kurzen persönlichen Vorstellungsrunde, und ich lerne, dass vor allem die Frauen mittleren Alters in meiner Gruppe glühende Fixer Upper-Fans sind. Zwei von ihnen haben die Reise nach Waco von ihren Ehemännern zum Hochzeitstag geschenkt bekommen, drei Mitvierzigerinnen sind auf einem „Girls Trip“ unterwegs. Nach der Vorstellungsrunde fragt uns Alyssa*, die die Tour führt, ob es okay sei, wenn wir mit einem Gebet begännen, denn Gott sei ein wichtiger Teil von Waco Tours. Was mich bei meiner ersten Tour-Teilnahme ziemlich irritiert, ${ }^{12}$ stört im Bus niemanden, und so

11 Waco Tours ist auf dem Bewertungsportal TripAdvisor registriert und bittet alle, die an einer Tour teilgenommen haben, dort eine Bewertung abzugeben. Im April 2021 hatte das Unternehmen 4074 Bewertungen erhalten, davon waren 4016 (98,5\%) ,,ausgezeichnet“, 48 ,sehr gut“, 11 ,,befriedigend“ und nur 2 ,mangelhaft“. https://www.tripadvisor.de/Attraction_Review-g56833-d10522669-Reviews-Waco_ Tours-Waco_Texas.html\#REVIEWS, zuletzt aufgerufen: 24. April 2021. Folgende Stimme kann exemplarisch für den Tenor der Bewertungen stehen: „My wife and I had a great time on this tour. At first we thought it might be a bit expensive, but afterwards we thought it was well worth it. We met the owner's and they were welcoming and friendly. All the staff was great! We felt like we'd been on a trip with our family. Our tour guides Matthew and Christina were fantastic! I highly recommend this tour." (Mann, ohne Ortsangabe, Mai 2019) (ebd.).

12 Ein Blick in die Foren von TripAdvisor zeigt, dass diese Irritation vereinzelt auch an anderer Stelle aufgetreten ist. So schreibt ein Mann aus New Jersey im Mai 2017: „Dear Waco Tour Owners, I have a personal right to be free of your religious beliefs. Yes, I know, things are different in Texas and parts of the South and Middle, but still ....“. Den Beginn der Tour beschreibt er wie folgt: ,The guides who were settled into their front seats, turned back toward the passengers, introduced themselves and then one of them said that she is instructed to say a prayer before commencing with the tour. Without pause she then began to pray, and heads were bowed. In that instant I suddenly felt an inner panic and my eyes darted for the exit wanting to bolt.“ (ebd.). Luke Whyte, der Mitbegründer von Waco Tours, antwortet wie folgt: „We are truly sorry 
segnet Alyssa* uns, die Tour und Waco, und wir machen uns auf den Weg: durch die vom Wirbelsturm zerstörte Innenstadt, vorbei an den Silos, über den Campus der Baylor University mit dem neuen McLane Football Stadium, hinüber nach East Waco, wo viele verfallene Häuser, aber auch eine bunte Stadtteilbibliothek zu sehen sind, durch den hügeligen Cameron Park und vorbei an einigen Fixer UpperHäusern. Hier und da steigen wir aus, um Fotos zu machen. Die Motive (Häuser, Landschaften, sich ins Bild schiebende Logos, ein wie zufällig vor seinem Laden herumstehender Fixer Upper-Protagonist ${ }^{13}$ ) sind so gewählt, dass sie sich für Selfies und Instagram-Posts hervorragend eignen. Zur Stärkung gibt es zwischendurch eine Portion Texas Pecan Ice Cream (lokale Spezialität) und eine Dr. Pepper Cola (deren Rezeptur in Waco erfunden wurde).

Als wir durch North Waco fahren, erzählt uns Alyssa*, dass sich bis vor Kurzem nicht einmal der Pizza-Service in diesen Teil der Stadt getraut habe, so gefährlich sei es hier gewesen (vgl. Abb. 9). Dann macht sie uns auf ein neues Lebensmittelgeschäft aufmerksam, das von Mission Waco, einer christlichen Wohltätigkeitsorganisation, eröffnet und von Chip und Joanna Gaines finanziert worden sei. Schließlich hält die Tour vor dem einzigen Fixer Upper-Haus an, das jemals für einen alleinstehenden Mann (Bachelor) renoviert wurde, einem, wie wir hören, Ex-Model. Ungeeignet für die oberflächliche Model-Welt der großen Metropolen sei der junge Texaner tief gefallen, bis er schließlich Jesus gefunden habe, nach Texas zurückgekehrt sei - und 2016 Waco Tours gegründet habe. Die Geschichte, die hier erzählt wird, ist die von David Ridley, der heute nicht nur CEO von Waco Tours ist, sondern auch Mitglied der Antioch Community Church in North Waco - an der wir gerade vorbeifahren. Sein Geschäftspartner Luke Whyte ist dort sogar College-Pastor. Und wie sich herausstellt, ist auch Chase*, unser Busfahrer, Pastor bei Antioch. Freudig erzählt er, dass er an seinen wenigen freien Tagen gerne Besucher wie uns durch Waco führe. Chase* und Alyssa* betonen, dass Antioch viel Gutes bewirkt habe in North Waco, von Bildungsprogrammen für benachteiligte Kinder bis zur Resozialisierung ehemaliger Zwangsprostituierter. Kurz wird die Sozialstruktur der Stadt problematisiert, denn gut ein Viertel der Wacoans lebt unterhalb der Armutsgrenze (United States Census Bureau 2020), aber es wird auch deutlich, dass es die christlichen Institutionen sind, die aus Sicht von Waco Tours zur erfolgreichen Bearbeitung dieser Probleme beitragen. Man hätte beispielsweise jedoch auch auf die lokalen Anlaufstellen von Planned Parenthood (wo u. a. Menschen beraten werden, die sexuell missbraucht wurden) oder Habitat for Humanity (einer NGO, die unabhängig von

that you felt trapped and uncomfortable because of the prayer at the beginning of our tour. Our intention is never to impose our beliefs on any of our guests. Out of our thousands of tours this is the first time we've gotten negative feedback on this specific aspect of our tour. But because of your experience, we will be adjusting our approach in how we include the prayer. Thank you for your feedback and again, we apologize that you were uncomfortable." (https://www.tripadvisor.de/Attraction_Review-g56833-d10522669Reviews-Waco_Tours-Waco_Texas.html\#REVIEWS, zuletzt aufgerufen: 24. April 2021).

13 Das Zusammentreffen mit Jimmy Don, der in Fixer Upper die Metallarbeiten für Joanna Gaines durchführt und den Fans der Show daher bestens bekannt ist, wird während der Tour als außergewöhnlicher Glücksfall inszeniert. Don steht vor seinem Geschäft und unterhält sich mit Leuten, der Tourbus kommt vorbei, hält abrupt - weil man Don erspäht hat - an, es gibt ein großes Hallo und die Gelegenheit zum Gruppenselfie. Die Inszenierung dieses „Zufalls“ fiel mir freilich erst bei meiner zweiten Tourteilnahme auf. Die Szene wird auch in journalistischen Texten über Waco beschrieben (Petersen 2019). 
der religiösen Orientierung eines Menschen menschenwürdige Wohnungen baut) hinweisen können. Zwar sprechen Chase* und Alyssa* keine explizite Einladung an uns aus, am Sonntag zu den Gottesdiensten von Antioch zu kommen oder über eine mögliche christliche Wende unseres Lebens nachzudenken, doch verdichtet sich in mir immer stärker das Gefühl, eher einer zeitgenössisch-populären, wenngleich weitgehend implizit bleibenden Evangelisierungsveranstaltung als einer Stadtführung beizuwohnen. Zwar erhält die Stadtführung durch das Gebet gleich zu Beginn eine religiöse Rahmung und informiert später ausführlich über die Sozialprogramme der Antioch Church, viel eindrucksvoller aber ist die Tour als Erlebnisformat. Chase* und Alyssa* vermitteln uns mit großem jugendlichem Enthusiasmus, dass für sie in den erneuerten Häusern und den vielfältigen Zeichen christlicher Wohltätigkeit das Wirken Gottes unmittelbar sinnlich erfahrbar sei und dass sie auch uns, ihre Gäste, die sie wie Freunde oder Familienmitglieder behandeln, an dieser Erfahrung teilhaben lassen wollen.

Etwas abstrakter betrachtet wird auch hier die erzählte Stadtgeschichte unterteilt in eine ,Zeit davor“", einen „Wendepunkt“ und eine ,Zeit danach“. Die thematische Gattungsstruktur der Konversionserzählung (Ulmer 1998) idealtypisch abbildend füllen die Tour-Guides die „Zeit davor“ mit Darstellungen von Krise und Niedergang. Hauptbezugspunkte sind die Zerstörung der Innenstadt durch den Tornado im Jahr 1953 und der Imageschaden, den Waco durch die „Waco Siege“ im Jahr 1993 erlitten hat. Völlig unerwähnt bleiben hingegen die zutiefst rassistischen Facetten der Stadtgeschichte mit Plantagenwirtschaft und Sklavenhaltung im 19. Jahrhundert (wofür die Silos als einstige Baumwollsamendepots geradezu ikonisch stehen) oder mit den Lynchmorden und dem Wirken des Ku Klux Klan Anfang des 20. Jahrhunderts. Man kann diese Form der Geschichtsdarstellung durchaus als „Whitewashing“ bezeichnen: Waco erscheint eher als Opfer von Naturgewalt und schicksalhaften äußeren Zuschreibungen, denn als Ort eigener teils grausamer Handlungen (von Weißen). Der „Wendepunkt“" zum Besseren verdichtet sich in den Erzählungen von Waco Tours zwar auch im TV-Erfolg von Chip und Joanna Gaines (wohl auch, weil sich die Touristen genau dafür interessieren), wird aber in der Gesamtinszenierung komplexer angelegt - und zwar als heilendes Zusammenspiel von Fixer Upper, Magnolia und der Antioch Community Church.

Die „Zeit danach“ vermittelt sich als etwas uneingeschränkt Positives, das allerdings erst im Entstehen begriffen ist, was es nicht weniger faszinierend, weil umso transzendenter macht. Waco befindet sich, so der Tenor, an der Schwelle zu etwas Besserem, und es ist diese Übergangsphase, die auf den Touren thematisch wird. Chase* und Alyssa* heben das ungeheure Potenzial dieses sich vollziehenden Wandels hervor, wovon gerade die noch nicht renovierten Häuser der Stadt zeugen (vgl. Abb. 9). Sie lesen den Wandel als unmittelbares, wenngleich durch ihr eigenes Tun zu unterstützendes Wirken Gottes im Raum der Stadt - was charakteristisch für evangelikale Weltdeutungen ist, allerdings auch eine Reihe von erkenntnistheoretischen Problemen mit sich bringt, für die der gebaute Raum eine Lösung bereithält: Da sie Gott als Person oder Freund imaginieren, der kausal sowohl in das eigene Bewusstsein als auch in die Wirklichkeit der Alltagswelt eingreifen kann, stehen Evangelikale permanent vor einer doppelten kognitiven Herausforderung. Auf der Ebene des Bewusstseins gilt es, die eigenen Gedanken, Wünsche, Begehren und 
Pläne von der Wahrnehmung göttlicher Botschaften, die in Form spontaner Assoziationen und Bilder, aber auch als konkrete Handlungsanweisungen unvermittelt im Bewusstsein auftauchen können, zu unterscheiden (Luhrmann 2012). Auf der Ebene der mit anderen geteilten Alltagswirklichkeit, in der sich aus evangelikaler Sicht nichts zufällig ereignet, sondern alles, was passiert, das Resultat eines Kräftemessens zwischen guten (Gott) und bösen (Satan) Kräften ist, besteht die Herausforderung darin, das göttliche Wirken zu erkennen und als Teil eines nicht immer sofort durchsichtigen göttlichen Planes zu verstehen, um diesem Plan durch das eigene Handeln zum Sieg über das Böse zu verhelfen. Für dieses hermeneutische Problem entwickeln Evangelikale allerlei kognitive Strategien (Steets 2020), wobei der gebaute Raum als Evidenzstifter für das göttliche Wirken fungiert. Dies lässt sich phänomenologisch erklären: So wissen wir von Alfred Schütz, dass , , $[n]$ ur Erfahrungen von physischen Dingen innerhalb der manipulativen Zone [...] den grundlegenden Test aller Wirklichkeit, nämlich den Widerstand [erlauben]“ (Schütz 2003, S. 199). Das heißt, Menschen erfahren diejenigen Dinge, Räume oder Lebewesen am wirklichsten, die sich in ihrem Hier und Jetzt befinden, die sie anfassen und unmittelbar sinnlich wahrnehmen können. Das löst zwar nicht das grundlegende hermeneutische Problem (,Wer wirkt hier eigentlich gerade?“), verschafft aber der in evangelikalen Gemeinschaften kommunikativ erzeugten Antwort (,Es ist Gott!“) eine enorme Plausibilität. Materielle Räume verwirklichen damit in sehr konkretem Sinne die religiös imaginierte symbolische Ordnung der Stadt. Dem starken evangelikalen Missionierungsbedürfnis folgend gilt es, diese Ordnung auch für Außenstehende lesbar zu machen. Dafür wiederum eignen sich touristische Erlebnisformate ganz hervorragend, denn auch sie spielen mit der Suche nach der Wirklichkeit hinter den Bildern und Zeichen. Anders formuliert: Der Tourismus ist geprägt von dem Versprechen, etwas hautnah, multisinnlich und vor Ort zu erleben, um das „Authentische“ hinter den Bildern und Zeichen zu entdecken (Schäfer 2015), auch wenn dies nur allzu oft inszeniert ist (MacCannell 1973). Aus evangelikaler Sicht wiederum sind es ähnlich starke Sinneseindrücke, durch die Gottes Wirken unmittelbar erkennbar ist. So entsteht auf der Erlebnisebene eine Schnittstelle zwischen Tourismus und Evangelikalismus - für die die Antioch Community Church eine vermittelnde Schlüsselrolle einnimmt.

\subsection{Die Ästhetisierung der Missionierungskommunikation}

Die Antioch Community Church von Waco spaltete sich in den 1990er-Jahren von der Highland Baptist Church ab, einer der großen Baptistengemeinden der Stadt. Bei Highland blieben eher die traditionellen Familien, während - wie ein ehemaliges Mitglied von Highland es beschreibt - „Antioch always felt young and, like, sexy“ (zit. in Petersen 2019). Heute hat Antioch Waco rund 5000 Mitglieder, die nicht ausschließlich, aber doch mehrheitlich Weiße sind - unter ihnen viele, die bei Magnolia oder Waco Tours arbeiten. 1999 zog Antioch in einen alten HEB-Lebensmittelladen in North Waco, einem Stadtviertel, das damals aus Sicht der (weißen) Mittelschicht niemand freiwillig betrat. Antioch machte es sich zur Aufgabe, eben jene „leidende“ Nachbarschaft im göttlichen Geiste zu erneuern. Allgemein beschrieben hört sich das so an: „[W]e plant churches and impact communities with God's love so we 
might see His Kingdom come and His will be done“ (Antioch Community Church 2021). ${ }^{14}$ Konkret auf Waco bezogen heißt das:

We believe church doesn't just happen on Sundays. In the Bible, the first church was commissioned to live in community, to worship, to share meals, to praise God together and to reach out to others. We live out this mission daily here in Waco, in coffee shops, church pews, on soccer fields and street corners. [...] WE WILL BE PART OF TRANSFORMING WACO, TEXAS. We will serve and reach out, both through specific church ministries and in our day-to-day lives (ebd., Hervorhebung im Original).

Betont wird die breite Alltagsrelevanz des christlichen Lebens, wobei Antioch bestrebt ist, die biblische Zielstellung, ,in Gemeinschaft zu leben, Gottesdienst zu feiern, Mahlzeiten zu teilen, Gott gemeinsam zu loben und auf andere zuzugehen“ (ebd, eigene Übersetzung) in zeitgenössische Formate zu übersetzen, wie sich besonders am Beispiel der Missionierung zeigt. Als Antioch anfing, in North Waco zu missionieren, versuchte man es zunächst mit den althergebrachten Formen der Evangelisation - wie Straßenpredigten und dem Tingeln von Haustür zu Haustür, um Menschen in ein Gespräch über Jesus zu verwickeln. Dies erwies sich jedoch als wenig effektiv (Petersen 2019). Wie andere moderne evangelikale Megakirchen auch versuchte Antioch schließlich, die konservative Grundhaltung, die eine große Bibeltreue mit dem Bild eines grundsätzlich sündigen, aber rettbaren Menschen verbindet, mit einer zeitgemäßen Ästhetik und einem modernen Erlebnischarakter zu vereinen (Wellman et al. 2020), um weniger aus der Zeit gefallen und weniger antagonistisch gegenüber der säkularen und kapitalistischen Welt zu erscheinen. Architektur und der gebaute Raum begannen für diese Neuausrichtung eine entscheidende Rolle zu spielen. Neben der Kirche zogen auch Teile des pastoralen Personals und Dutzende Mitglieder nach North Waco (Petersen 2019) - wo sie sich, zunächst von einer kleinen Hausrenovierungsfirma zweier Gemeindemitglieder namens Chip und Joanna Gaines und später international beachtet in der TV-Show Fixer Upper, ihre günstig erworbenen Häuser ästhetisch höchst zeitgemäß im Stile des „Farmhouse Chic“ renovieren ließen. Da sich Antioch die Modernisierung biblisch verbriefter Formen christlichen Lebens auf die Fahnen geschrieben hat, verwundert es nicht, dass innerkirchlich diese Renovierungen von Beginn an als spirituelle Erneuerung und als Beitrag zur „Heilung“ Wacos verstanden wurden (Petersen 2019). Ähnlich dürfte es sich mit den Stadtführungen von Waco Tours verhalten, die den Wandel auch für nichtevangelikale Augen als göttliches Wirken lesbar machen (sollen) und damit nicht unwesentlich zur Institutionalisierung der symbolischen Neuordnung der Stadt beitragen.

Dies hatte verschiedene Effekte: Chip und Joanna Gaines avancierten im Zuge des Erfolges von Fixer Upper geradezu zur idealtypischen Verkörperung einer neuen popkulturell anschlussfähigen Version des Evangelikalismus - und nebenbei wird klar, warum so viele Hausbesitzer aus Fixer Upper Mitglieder der Antioch Commu-

14 Die Antioch-Bewegung mit ihrem Ursprung 1987 und Hauptsitz in Waco hat mittlerweile ein globales Netzwerk aufgebaut, das 45 Kirchen (,planted churches“) in den USA und über 80 Missionsstationen in der ganzen Welt umfasst (https://antioch.org/our-story/, zuletzt aufgerufen: 24. April 2021). 
nity Church sind (vgl. Fußnote 4). Auf lokaler Ebene verband sich eine Mitgliedschaft bei Antioch bald mit der Vorstellung, Teil der sozialen und wirtschaftlichen Aufschwungserzählung Wacos zu sein, also an etwas Fortschrittlichem und Trendigem teilzuhaben, das sich gleichzeitig als höchst lukrativ erwies und völlig nahtund reibungslos mit dem Leben wiedergeborener Christen vereinbar war. Die Gründer von Waco Tours verkörpern diese Geschichte auf ähnlich idealtypische Weise wie Chip und Joanna Gaines. Auch den Whytes und Ridleys ist es gelungen, die christliche Botschaft mit einer perfekten jugendlich-familiären Ästhetik und zeitgenössischen Formaten der Popkultur zu verschmelzen und dabei ökonomisch höchst erfolgreich zu sein. Auf ihren Stadtführungen wird der gebaute Raum mit seinen vielfältigen phänomenologischen Erlebnisqualitäten zur ,materiellen Objektivation“ (Knoblauch und Steets 2020) und damit zur zentralen Vermittlungsinstanz für eine neue Form der religiösen Kommunikation (Knoblauch 2009, S. 68; 2017). Das zeigt sich am deutlichsten in den beschriebenen Formen der Missionierung: Missionierung funktioniert kaum noch explizit über sprachliche Botschaften, sondern sehr viel impliziter über die kreative Gestaltung von Räumen, verbunden mit der Produktion von Narrativen und Imaginationen über den Wandel dieser Räume, die als Legitimationen (Berger und Luckmann 1970) fungieren. Eine solche ästhetisierte Form der Missionierungskommunikation erinnert - wenngleich inhaltlich völlig anders gelagert - stark an das, was seit den 1990er-Jahren als „Kulturalisierung der Stadt“ (Reckwitz 2012, S. 269ff.) beforscht und diskutiert wird. Städte, so die zugrundeliegende These, werden zu Orten der beständigen Produktion und Vermarktung von Zeichen, Lebensstilen, Erlebnisqualitäten und Atmosphären und damit zu Schlüsselorten eines spätmodernen kulturellen Kapitalismus (ebd; Zukin 1995). Indem sich der Evangelikalismus dieser Ästhetisierungsstrategien bedient, macht er sich genau daran unmittelbar anschlussfähig.

\section{Schluss: Populäre Religion, das Sentimentale und die Refiguration von Räumen}

Die Befunde der empirischen Analyse legen es nahe, den beschriebenen Wandel Wacos über das Zusammenspiel von Raum und Religion zu erklären. Resultat dieses Zusammenspiels, so meine These, ist eine neue popkulturell-touristische Sozialform des Religiösen, die unterschiedliche Formate annehmen kann - von der TVHausrenovierungsshow bis zum touristischen Erleben städtischer Räume - und die geprägt ist erstens durch ein evangelikales Erneuerungsnarrativ, zweitens durch die rituelle Herstellung und das materielle Wirklichwerden einer symbolischen Neuordnung von Räumen und drittens durch die Ästhetisierung der evangelikalen Missionierungskommunikation. Diese popkulturell-touristische Sozialform des Religiösen geht nicht nur einher mit einem erstaunlichen Imagewandel der Stadt Waco, sondern ist geradezu dessen Motor. Ein stärker abstrahierender Blick auf diesen Wandel macht deutlich, dass Raum und Religion hier offenbar in einem wechselseitigen Bedingungsverhältnis stehen: Einerseits wird der Raum zum zentralen Medium der religiösen Kommunikation, andererseits fungiert die Religion als identitätsstiftender Bezugspunkt spätmoderner Raumkonstruktionen. Um dieses Zusammenspiel zu 
verstehen, möchte ich abschließend die identifizierte popkulturell-touristische Sozialform des Religiösen noch einmal stärker sozialtheoretisch durchleuchten und mit der Idee des Sentimentalen verbinden, um daran anknüpfend ihr besonderes Place Making-Potenzial herauszustellen. Wacos Wandel deute ich schließlich gesellschaftsdiagnostisch als „Refiguration von Räumen“ (Löw und Knoblauch 2021).

\subsection{Kommunikative Konstruktion, Raum und Religion}

Religionssoziologisch schließt meine Analyse an Arbeiten von Hubert Knoblauch (2009) und Thomas Luckmann (1991) an. Während Luckmann noch davon ausgegangen war, dass religiöse Inhalte, wenn sie in nichtreligiösen sozialen und kommunikativen Formen auftreten, nicht mehr als Religion erkennbar sind und damit „unsichtbar“ werden, geht es Knoblauch darum, den Blick für solche neuen Sozialformen des Religiösen zu schärfen und ihre neue Art der Sichtbarkeit empirisch zugänglich zu machen. Dies setzt einen offenen Religionsbegriff voraus, den er über die Auseinandersetzung mit dem Begriff der Transzendenz herleitet (Knoblauch 2009, S. 53ff.). Kurz gesagt, Religion entsteht für Knoblauch immer dann, wenn Transzendenzerfahrungen, egal ob kleiner, mittlerer oder großer Art (Schütz und Luckmann 2003, S. 598ff.) nicht einfach nur bearbeitet, sondern kommunikativ sakralisiert werden. Sozialtheoretisch basiert diese Perspektive auf einer kommunikativen Erweiterung des Sozialkonstruktivismus (Knoblauch 2017), in deren Zentrum ein relationales Verständnis des Sozialen steht. Kommunikation - und das ist wichtig - kann also religiöse Formen annehmen, immer aber erzeugt sie Räume. Wie ist das zu verstehen?

Für Knoblauch ist jedes soziale Handeln ein kommunikatives Handeln, denn das Soziale wird bei ihm als Relation zwischen mindestens zwei Subjekten gefasst, die sich - und zwar immer vermittelt über „Objektivationen“ (Knoblauch 2017, S. 161ff.), das heißt für einander und für andere wahrnehmbar - wechselseitig aufeinander beziehen. Die Grundeinheit des Sozialen ist im einfachsten Fall eine Triade zwischen zwei Subjekten und einer Objektivation (ebd.: 112). Knoblauch betont, dass sowohl die Art und Weise, wie sich Subjekte aufeinander beziehen (ob sie gestikulieren oder sprechen, ob sie sich Aufmerksamkeit schenken oder sich ignorieren, ob sie einander anblicken oder sich schlagen etc.), als auch die Art und Weise, wie Objektivationen diese Bezugnahme vermitteln (also ob eher immateriell über Sprache oder eher materiell über Dinge und gebaute Räume, ob über analoge oder digitale Medien, ob zeichenhaft oder physisch wirkend), so breit wie nur irgend möglich zu denken sind. Gesellschaftstheoretisch zeigt er (ebd.: 189ff.), wie sich das so verstandene Soziale in unterschiedlichen gesellschaftlichen Zusammenhängen institutionalisiert, also wie aus losen kommunikativen Handlungen Handlungssequenzen, kommunikative Formen und Gattungen oder - wenn wechselseitige Bezugnahmen aufeinander sakralisiert werden - rituell-symbolische Ordnungen entstehen. Mit Blick auf die Räumlichkeit des Sozialen zeichnet er nach, wie aus ephemeren Verortungen und räumlichen Relationen feste räumliche Anordnungen und Raumsynthesen werden, die sich im Gebauten ebenso wie im Imaginären zu Mustern kristallisieren (zum Raum vgl. ausführlicher Knoblauch und Steets 2020). Diese Muster lassen sich wiederum zu idealtypischen Modi der Verräumlichung 
des Sozialen verdichten. Martina Löw (2020) schlägt vier distinkte Varianten vor, die sie „Raumfiguren“ nennt: Territorium, Netzwerk, Ort und Bahnenraum. Gesellschaftsdiagnostisch gehen Knoblauch und Löw (2021) davon aus, dass sich in der Gegenwart verschiedene Raumfiguren mit ihren unterschiedlichen Logiken der Verräumlichung spannungsreich überlagern - was Effekte zeitigt, die sie als „Refiguration von Räumen“ verstehen ${ }^{15}$. Während Territorien einer Logik der Grenzziehung und Homogenisierung im Rauminneren folgen und als flächenhaft imaginiert werden, verräumlicht das Netzwerk entlang einer Logik der Verknüpfung. Im Netzwerk sind realräumliche Distanzen irrelevant, was zählt, ist allein die je netzwerkspezifische Beziehung eines Elementes zu anderen Elementen. Bahnen wiederum folgen einer Logik der Durchquerung und damit eindeutiger als Netzwerke einer klassischmodernen instrumentellen Vernunft. Sie verbinden Start- und Zielpunkte (z. B. einer Reise oder einer Warenkette) miteinander und sind gebaut, um einen möglichst effizienten und reibungslosen Transport der zirkulierenden Elemente zu gewährleisten. Als Infrastrukturen können Bahnen (unbekannte) Räume erschließen oder grundlegend neu ordnen, wofür die USA ein sehr gutes Beispiel darstellen: Man denke an die Trails der Siedlerinnen, die Routen der Post, die Schienenwege der Eisenbahn - und nicht zuletzt an die Autobahntrassen des Interstate-Systems. Orte schließlich folgen einer Logik der Gleichzeitigkeit heterogener Bezüge. Die Art und Weise, wie an ihnen auf Geschichte und Welt referiert wird, wie sich die unterschiedlichsten ,thens and theres“ im ,here-and-now“ (Massey 2005, S. 140) verbinden und manifestieren, macht Ausschnitte auf der Erdoberfläche zu Orten (Berking 1998). Deshalb werden Orte als einzigartig imaginiert und haben Namen. Da die Spätmoderne das Besondere, das Einzigartige, ja Singuläre prämiert (Reckwitz 2017), werden heutzutage nicht nur Orte wichtiger, sondern auch Praktiken des Place Making und Formen der Verortung.

Die Diagnose der Refiguration überträgt Knoblauch (2020) auch auf die Religion, und zwar als spannungsreiche Gleichzeitigkeit von Säkularisierung und ReSakralisierung, von traditioneller Religiosität und neuer Spiritualität, von Be- und Entgrenzung der Religion, die er jeweils den gesellschaftlichen Großfigurationen von Moderne und Spätmoderne zuordnet. In der Spätmoderne, so seine Diagnose (Knoblauch 2009), werde mit der Verbreitung der Populären Religion das Subjekt und sein Inneres zum privilegierten und gefeierten Ort religiöser (Selbst-)Erfahrung - was für konservative Formen des Evangelikalismus ebenso gelte wie zum Beispiel für die alternative Spiritualität des New Age. Mit anderen Worten: Die Populäre Religion betont, evoziert und inszeniert in besonderer Weise die subjektive Erfahrung von Transzendenz. Genau dies beobachten wir auch in Waco. Hier gehen spätmoderne Formen der Religiosität/Spiritualität mit spätmodernen Formen der Produktion von Orten - also mit einer bestimmten Raumfigur - eine enge Symbiose ein. Im Fall der hier rekonstruierten popkulturell-touristischen Sozialform des Religiösen kommt, so meine These, dem Sentimentalen als Subjektivierungsmodus der Populären Religion

\footnotetext{
15 In der folgenden Darstellung der vier Raumfiguren beziehe ich mich auf die Vorarbeiten von Martina Löw und Hubert Knoblauch (Löw 2020; Löw und Knoblauch 2021), reichere deren Charakterisierung und Zusammenspiel aber mit eigenen Überlegungen an, die als Vorschläge zur Weiterentwicklung der Raumfiguren und der Refiguration zu verstehen sind.
} 
einerseits und als erlebnisbasierte Zuschreibung einer kleinstädtischen Ortsqualität andererseits eine bedeutende Rolle zu.

\subsection{Das Sentimentale und die Produktion von Orten}

Zwar stellt das Sentimentale in der Soziologie noch keine etablierte Begrifflichkeit dar, doch zeigen jüngere kulturwissenschaftliche Arbeiten (Ellis 1996; Kappelhoff 2004; Paul 2014) sein gesellschaftsanalytisches Potenzial. Soziologisch lässt sich das Sentimentale als beziehungsstiftender kommunikativer Code fassen, der eine gesteigerte Emotionalität und Sinnlichkeit in den wechselseitigen Bezugnahmen von Subjekten aufeinander (Kommunikation) und in den damit verbundenen Formen der gefühlsbasierten Subjektivierung und Identitätsbildung erzeugt. Das Sentimentale weist eine gewisse Nähe zum Affekt auf (Slaby und von Scheve 2019), adressiert aber weder einen spezifischen Affekt noch eine spezifische Emotion, sondern eher einen Modus des Fühlens. An der Schnittstelle von Individuum und Gesellschaft angesiedelt lässt es sich empirisch beobachten als eine aktive Hinwendung zu bzw. eine strategische Inszenierung oder ein Inszeniertsein von Emotionen, was wiederum affizierend auf das Subjekt zurückwirken kann. Es umfasst Phänomene wie das subjektive Schwelgen in Gefühlen (oder religiösen Transzendenzerfahrungen) ebenso wie besonders gefühlige (oder eine spirituelle Haltung fördernde) literarische, mediale und räumliche Formate. Das macht das Sentimentale zu einem spezifisch modernen Phänomen, denn es setzt erstens die Selbstreflexivität des Subjekts voraus, was zum Beispiel ein „Baden“ in der eigenen Traurigkeit oder Freude oder Spiritualität erst ermöglicht, und es basiert zweitens auf der Vorstellung, dass solche Gefühle und Emotionen gezielt, etwa im Kino, aber auch zum Beispiel über die räumliche Konstruktion von Atmosphären oder Formen des Urbanen (vgl. Thibaud 2011), hergestellt werden können. Das Sentimentale taucht, so vermute ich, verstärkt in Phasen gesellschaftlicher Umbrüche und Refigurationen auf, denn es scheint ein Versprechen auf ontologische Sicherheit (im Sinne einer ganzheitlichen Erfahrung von Emotionen) mitzuführen.

Im Falle Wacos ist das Sentimentale in der bereits angedeuteten Doppelrolle als Subjektivierungsmodus einerseits und als erlebnishafte Zuschreibung einer kleinstädtischen Ortsqualität andererseits zu beobachten. Das Imaginaire der amerikanischen Small Town ist weder ländlich noch kosmopolitisch-urban, sondern in erster Linie sentimental (Strauss 2017, S. 188ff.). Es ist verbunden mit der Vorstellung familiärer Herzlichkeit an öffentlichen Orten. Ray Oldenburg (1999) verdichtet dies im Begriff der „Great Good Places“, der „,informal public gathering places“ und „homes away from home“, wo Fremde aufeinandertreffen, sich dennoch zuhause fühlen und gerne verweilen (ebd.: xi). Wacos Place Making-Strategien orientieren sich in nahezu idealtypischer Weise an dieser Vorstellung: Die Stadt inszeniert sich als Ort, an dem weder Anonymität und Distanzierung noch große Hotelketten und Shopping Malls regieren, sondern kleine inhabergeführte Shops und Übernachtungsmöglichkeiten bei Freunden (Airbnbs). Hier zählen traditionelle kleinstädtische Werte wie Freundlichkeit, Familie, warmherzige Gastfreundschaft und eine Nähe zu Handwerk und Natur noch etwas; hier dürfen (weiße) Männer noch (weiße) Männer und (weiße) Frauen noch (weiße) Frauen sein und - ganz wichtig: Hier hat die Reli- 
Abb. 10 Ölstaat Texas: Benzinpreise in Waco, Texas (a) und Berkeley, Kalifornien (b). Fotos: Silke Steets, 2019
Abb. 11 „This is a sign“ Evangelikaler Humor vor einem trendigen Café in Waco. Foto: Silke Steets, 2019
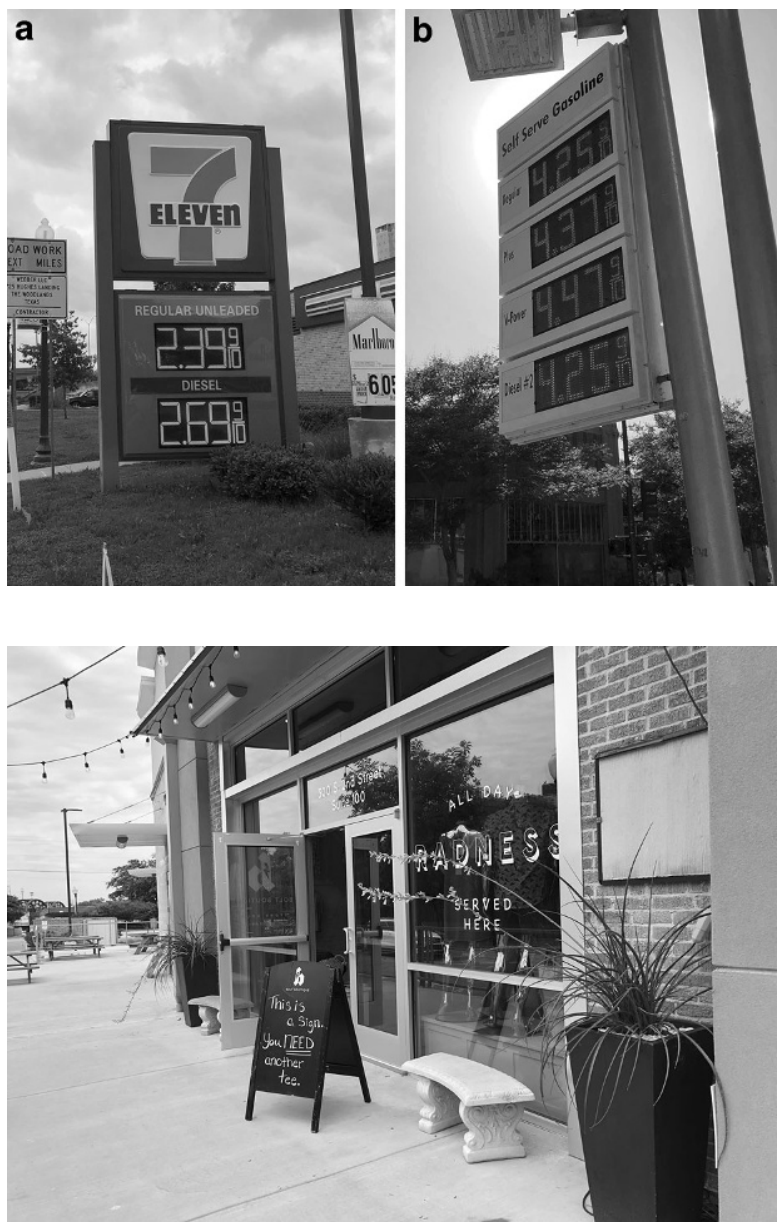

gion nicht nur eine völlig selbstverständliche Alltagsrelevanz, sondern kommt auch noch hip und zeitgemäß daher. Mittels der oben dargestellten touristischen Erlebnisformate lassen sich diese Qualitäten zudem im sentimentalen Subjektivierungsmodus verarbeiten, also dem Schwelgen in den Gefühlen und Identitätsangeboten, die die amerikanische Small Town und Waco im Besonderen ihren Besucherinnen macht.

Während in vielen US-Metropolen wie New York, San Francisco oder Dallas in den letzten Jahren Orte des informellen Herumhängens gezielt - vor allem in Form von oft privat bewirtschafteten Parks wie dem Bryant Park in Manhattan (Zukin 1995, S. 29ff.) oder dem Klyde Warren Park in Dallas - zur Wiederbelebung der Innenstädte erschaffen wurden, orientiert sich Chip und Joanna Gaines' Magnolia Market at the Silos räumlich-ästhetisch an genau diesen Vorbildern (vgl. Abb. 6). Interessant ist, dass eine solche zutiefst kleinstädtisch imprägnierte Form der Ortsproduktion über den Umweg der Metropolen - und damit gewissermaßen kosmopolitisch geadelt - wieder in der Small Town angekommen ist. Dort setzt sie Prozesse der kreativen Wiederbelebung in Gang, die selbst an die Urbanitätsvorstellungen 
der New Urban Middle Class anschlussfähig sind. Genau deshalb fühlt sich Waco heute auch für akademisch gebildete, liberale, subkulturelle Wacoans wie Kate* ein bisschen so an wie „Austin [...] in the early 80s“(Int_Kate*: Z. 773). Deutlich wird auch, dass der Erlebnismodus des Ortes ein multisinnlicher ist - womit wir beim spannungsreichen Gegensatz von Ort und Bahnenraum wären.

\subsection{Ort vs. Bahnenraum oder: Refiguration von Räumen}

Die in Waco zelebrierte Sozialform des popkulturell-touristischen Religiösen fungiert als zentraler Bezugspunkt der spätmodernen und sentimentalen Place MakingStrategien der Stadt. Die verschiedenen ,thens and theres“ von Small Town, Hinterland und Metropole, von Religion und Geschichte, die sich im „here-and-now“ manifestieren, macht Waco zu einem Ort und verhilft sowohl der Stadt wie der Religion zu neuer Sichtbarkeit. Dem allerdings steht noch immer die im Ölstaat Texas (vgl. Abb. 10) nahezu selbstevidente modern-instrumentelle Verräumlichungslogik des Bahnenraums in Form der Interstate-Autobahn gegenüber.

Wie eingangs bereits dargestellt, durchschneidet die I-35 Waco von Nordost nach Südwest und verbindet das ökonomische (Dallas/Fort Worth) mit dem politisch-kulturellen (Austin/San Antonio) Zentrum von Texas. In dieser Geographie ist Waco lediglich ein beliebiger ,stopover on the way up and down I-35“ (Petersen 2019). Ähnlich wie der Ort - und anders als die im Alltag abstrakt bleibenden Netzwerkoder Territorialräume - sind auch Bahnenräume sinnlich erfahrbar (Löw und Knoblauch 2021, S. 40). In ihrer Untersuchung des Strips von Las Vegas zeigen Denise Scott-Brown, Robert Venturi und Stephen Izenour (2007), dass die räumliche Form der amerikanischen Stadt der 1960er-Jahre (und das gilt für Texas vielerorts bis heute) maßgeblich durch die Wahrnehmungsweise des Bahnenraums geprägt ist - und zwar weil sie vorwiegend aus dem fahrenden Auto heraus erlebt wird. Diese „Er-fahrung“ von Raum produziert einen ,,automobilisierten Blicks“ (Stierli 2010, S. $149 \mathrm{ff}$.), der aus der Stadt eine Abfolge von gebauten Symbolen und sprachlichen Zeichen macht, die an den Rändern des Bahnenraums um die Aufmerksamkeit der Vorbeifahrenden werben (vgl. Abb. 2). Zwar unterscheiden sich die Zeichen und Symbole in der Art, in der sie ihre Botschaften kommunizieren - so gibt es „Enten“, also Gebäude, deren Sinn sich über ihre skulpturale Form vermittelt, und ,dekorierte Schuppen", also Gebäude, die so generisch sind, dass sie eine Beschriftung brauchen (Venturi et al. 2007, S. 106/107) -, der gebaute und gegliederte Raum selbst aber ist aus dem Auto heraus immer ein zeichenhaft hergestellter (Steets 2015, S. 189ff.).

Während in Waco mit dem Bahnenraum (zeichenhaft - modern - instrumentell) und dem Ort (multisinnlich - spätmodern - erlebnisbasiert) zwei unterschiedliche Raumfiguren mit ihren jeweiligen Wahrnehmungsweisen und Logiken die Verräumlichung des Sozialen vorantreiben, entsteht das, was Löw und Knoblauch (2021) als „Refiguration von Räumen“ bezeichnen. Das Ergebnis der spannungsreichen Überlagerung von Ort und Bahnenraum sind die neuen Raumanordnungen der coolen evangelikalen Urbanität. Neben einem hippen Café, das humorvoll ebenso mit der zeichenhaften Wahrnehmungslogik des (modernen) Bahnenraums wie mit dem hermeneutischen Problem des Evangelikalismus spielt (vgl. Abb. 11), sind die popkulturell-touristische Sozialform des Religiösen und die damit verbundenen Formen 
der Ästhetisierung von Räumen und Kommunikationsformen die zentralen Beispiele. Die sich hier abzeichnende Refiguration der Stadt hat vor allem eins bewirkt: dass Waco neu sichtbar wurde in der imaginären Geographie der USA. Und diese neue Sichtbarkeit ist es auch, die zum wachsenden Selbstbewusstsein des evangelikalen Christentums in den Vereinigten Staaten beiträgt (vgl. Fitzgerald 2017).

Danksagung Die diesem Artikel zugrundeliegenden Feldaufenthalte in Waco (Texas) wurden im Rahmen eines Heisenberg-Stipendiums realisiert und von der Deutschen Forschungsgemeinschaft finanziert (Projektnummer 326140490). Der SFB 1265 „Re-Figuration von Räumen“ hat die vertiefenden Recherchen zu Politik, Ökonomie und Geschichte Wacos finanziell unterstützt (Projektnummer 290045248). Für die ebenso kreative wie akribische Durchführung dieser Recherchen und die kritische Lektüre des Textes danke ich herzlich Nadine Schabét. Für wertvolle Kommentare, Nachfragen und Anregungen danke ich außerdem Meike Haken, Joanna Kiefer, Hubert Knoblauch, Kornelia Sammet sowie den anonymen Gutachterinnen.

Funding Open Access funding enabled and organized by Projekt DEAL.

Open Access Dieser Artikel wird unter der Creative Commons Namensnennung 4.0 International Lizenz veröffentlicht, welche die Nutzung, Vervielfältigung, Bearbeitung, Verbreitung und Wiedergabe in jeglichem Medium und Format erlaubt, sofern Sie den/die ursprünglichen Autor(en) und die Quelle ordnungsgemäß nennen, einen Link zur Creative Commons Lizenz beifügen und angeben, ob Änderungen vorgenommen wurden.

Die in diesem Artikel enthaltenen Bilder und sonstiges Drittmaterial unterliegen ebenfalls der genannten Creative Commons Lizenz, sofern sich aus der Abbildungslegende nichts anderes ergibt. Sofern das betreffende Material nicht unter der genannten Creative Commons Lizenz steht und die betreffende Handlung nicht nach gesetzlichen Vorschriften erlaubt ist, ist für die oben aufgeführten Weiterverwendungen des Materials die Einwilligung des jeweiligen Rechteinhabers einzuholen.

Weitere Details zur Lizenz entnehmen Sie bitte der Lizenzinformation auf http://creativecommons.org/ licenses/by/4.0/deed.de.

\section{Literatur}

Antioch Community Church. 2021. About. https://antiochwaco.com/about/. Zugegriffen: 23. Apr. 2021.

Augé, Marc. 1994. Nicht-Orte. Vorüberlegungen zu einer Ethnologie der Einsamkeit. Frankfurt a.M.: S. Fischer.

Barth, Lauren. 2020. Here's how much chip and Joanna Gaines are really worth. https://www.thelist.com/ 220944/heres-how-much-chip-and-joanna-gaines-are-really-worth/. Zugegriffen: 24. März 2021.

Berger, Peter L., und Thomas Luckmann. 1970. Die gesellschaftliche Konstruktion der Wirklichkeit. Frankfurt a.M.: Fischer.

Berking, Helmuth. 1996. Schenken. Zur Anthropologie des Gebens. Frankfurt a.M., New York: Campus.

Berking, Helmuth. 1998. „Global Flows and Local Cultures“. Über die Rekonfiguration sozialer Räume im Globalisierungsprozeß. Berliner Journal für Soziologie 8(3):381-392.

Bernstein, Patricia. 2005. The first Waco horror: the lynching of Jesse Washington and the rise of the NAACP. College Station: Texas A\&M University Press.

Burke, Anabel. 2018. The Ku Klux Klan in Waco. https://wacohistory.org/items/show/200. Zugegriffen: 14. Febr. 2021

Carrigan, William D. 2004. The making of a lynching culture: violence and vigilantism in Central Texas, 1836-1916. Urbana: University of Illinois Press.

Knoblauch, Hubert und Silke Steets. 2020. Von der Konstitution zur kommunikativen Konstruktion von Raum. In Grenzen der Kommunikation - Kommunikation an den Grenzen, Hrsg. Jo Reichertz, 134-148. Weilerwist: Velbrück.

Conger, Roger N. 2016. Waco, TX. https://www.tshaonline.org/handbook/entries/waco-tx. Zugegriffen: 12. Febr. 2021.

Douglas, Mary. 1985. Reinheit und Gefährdung: Eine Studie zu Vorstellungen von Verunreinigung und Tabu. Berlin: Dietrich Reimer. 
Ellis, Markman. 1996. The politics of sensibility: race, gender, and commerce in the sentimental novel. New York: Cambridge University Press.

Elwert, Frederik, Martin Redermacher, und Jens Schlamelcher (Hrsg.). 2017. Handbuch Evangelikalismus. Bielefeld: transcript.

Fitzgerald, Frances. 2017. The Evangelicals: the struggle to shape america. New York: Simon \& Schuster.

Florida, Richard. 2002. The rise of the creative class: and how it's transforming work, leisure, community and everyday life. New York: Basic Books.

Geertz, Clifford. 1987. Dichte Beschreibung. Beiträge zum Verstehen kultureller Systeme. Frankfurt a.M.: Suhrkamp.

Heft, Erin. 2019. High hotel occupancy, tourists are leading to more short term rentals in Waco, but not everyone is happy. https://www.kxxv.com/hometown/mclennan-county/waco-neighborhoods-reactto-vacation-rentals. Zugegriffen: 24. März 2021.

Jakle, John A. 2010. Paving America for the automobile. In The making of the American landscape, Hrsg. Michael P. Conzen, 403-422. London, New York: Routledge.

Kappelhoff, Hermann. 2004. Matrix der Gefühle: Das Kino, das Melodrama und das Theater der Empfindsamkeit. Berlin: Vorwerk.

Kiefer, Joanna Katherina. 2020. „Was gibt's für ein schöneres Geschenk und wir brauchen es nur annehmen “ - Die Struktur persönlicher Gottesbeziehungen im evangelikalen Milieu. Unveröffentlichte Masterarbeit. Universität Leipzig.

Knoblauch, Hubert. 2009. Populäre Religion: Auf dem Weg in eine spirituelle Gesellschaft. Frankfurt a.M., New York: Campus.

Knoblauch, Hubert. 2017. Die kommunikative Konstruktion der Wirklichkeit. Wiesbaden: Springer VS.

Knoblauch, Hubert. 2020. Einleitung: Die Refiguration der Religion. In Die Refiguration der Religion. Perspektiven der Religionssoziologie und der Religionswissenschaft, Hrsg. Hubert Knoblauch, 7-28. Weinheim: Beltz Juventa.

Landry, Charles. 2000. The creative city: a toolkit for urban innovators. London: Earthscan Publications.

Lawson, Drake. 2020. Waco: new poll on gentrification sparks conversation among residents. https://www. kwtx.com/content/news/Waco-New-poll-on-gentrification-sparks-conversation-among-residents567685661.html. Zugegriffen: 24. März 2021.

Löw, Martina. 2020. In welchen Räumen leben wir? Eine raumsoziologisch und kommunikativ konstruktivistische Bestimmung der Raumfiguren Territorialraum, Bahnenraum, Netzwerraum und Ort. In Grenzen der Kommunikation. Kommunikation an den Grenzen, Hrsg. Jo Reichertz, 149-164. Weilerwist: Velbrück.

Löw, Martina, und Hubert Knoblauch. 2021. Raumfiguren, Raumkulturen und die Refiguration von Räumen. In Am Ende der Globalisierung: Über die Refiguration von Räumen, Hrsg. Martina Löw, Volkan Sayman, Jona Schwerer, und Hannah Wolf, 25-57. Bielefeld: transcript.

Luckmann, Thomas. 1991. Die unsichtbare Religion. Frankfurt a.M.: Suhrkamp.

Luhrmann, Tanya M. 2012. When god talks back: understanding the American evangelical relationship with god. New York: Alfred A. Knopf.

MacCannell, Dean. 1973. Staged authenticity: arrangements of social space in tourist settings. American Journal of Sociology 79(3):589-603.

Magnolia Network. 2020. Press release: Chip and Joanna Gaines' Magnolia network announces return of hit series ,fixer upper,“ expands original programming slate. https://press.discovery.com/ us/magnolia-network/press-releases/2020/fixer-upper-reboot-announcement/. Zugegriffen: 23. Apr. 2021.

Massey, Doreen B. 2005. For space. London, Thousand Oaks: SAGE.

Oldenburg, Ray. 1999. The great good place. Cafés, coffee shops, bookstores, bars, hair salons, and other hangouts at the heart of community. New York: Marlowe.

Paul, Heike. 2014. The myths that made america. Bielefeld: transcript.

Petersen, Anne Helen. 2019. „Fixer upper“ is over, but Waco's transformation is just beginning. https:// www.buzzfeednews.com/article/annehelenpetersen/waco-texas-magnolia-fixer-upper-antioch-chipjoanna-gaines. Zugegriffen: 24. März 2021.

Ray, Skylar. 2019. I-35. https://wacohistory.org/items/show/179. Zugegriffen: 14. Febr. 2021.

Reckwitz, Andreas. 2012. Die Erfindung der Kreativität: Zum Prozess gesellschaftlicher Ästhetisierung. Berlin: Suhrkamp.

Reckwitz, Andreas. 2017. Die Gesellschaft der Singularitäten. Zum Strukturwandel der Moderne. Berlin: Suhrkamp.

Sachs, Andrea. 2020. The Magnolia effect. https://www.pressreader.com/canada/vancouver-sun/20200201/ 283897344986218. Zugegriffen: 23. Apr. 2021. 
Schäfer, Robert. 2015. Tourismus und Authentizität: Zur gesellschaftlichen Organisation von Außeralltäglichkeit. Bielefeld: transcript.

Schütz, Alfred. 2003. Über die mannigfaltigen Wirklichkeiten. In Theorie der Lebenswelt 1: Die pragmatische Schichtung der Lebenswelt, Bd. V.1, Hrsg. Martin Endreß, Ilja Srubar, 181-239. Konstanz: UVK.

Schütz, Alfred, und Thomas Luckmann. 2003. Strukturen der Lebenswelt. Konstanz: UVK.

Slaby, Jan, und Christian von Scheve. 2019. Affective societies. London, New York: Routledge.

Smith, Brent L., Kelly R. Damphousse, und Paxton Roberts. 2006. Pre-incident indicators of terrorist incidents: the identification of behavioral, geographic, and temporal patterns of preparatory conduct. https://www.ncjrs.gov/pdffiles1/nij/grants/214217.pdf. Zugegriffen: 14. Febr. 2021.

Steets, Silke. 2005. Doing Leipzig. Räumliche Mikropolitiken des Dazwischen. In Die Wirklichkeit der Städte. Hrsg. Helmuth Berking und Martina Löw, Martina, 107-121. Soziale Welt Sonderband 16. Baden-Baden: Nomos.

Steets, Silke. 2015. Der sinnhafte Aufbau der gebauten Welt: Eine Architektursoziologie. Berlin: Suhrkamp.

Steets, Silke. 2020. Kognitive Minderheiten in Leipzig und Dallas: Zur Aktualisierung eines religionssoziologischen Konzepts von Peter L. Berger. In Die Refiguration der Religion. Perspektiven der Religionssoziologie und der Religionswissenschaft. Hrsg. Hubert Knoblauch, 129-146. Weinheim: Beltz-Juventa.

Stierli, Martino. 2010. Las Vegas im Rückspiegel: Die Stadt in Theorie, Fotografie und Film. Zürich: gta.

Strauss, Anselm L. 2017. Images of the American city. London, New York: Routledge.

The Waco History Podcast. 2019. East Waco: past, present, and future. https://wacohistorypodcast.com/ east-waco/. Zugegriffen: 24. März 2021.

Thibaud, Jean-Paul. 2011. The sensory fabric of urban ambiances. The Senses and Society 6(2):203-215.

Ulmer, Bernd. 1998. Konversionserzählungen als rekonstruktive Gattung: Erzählerische Mittel und Strategien bei der Rekonstruktion eines Bekehrungserlebnisses. Zeitschrift für Soziologie 17(1):19-33.

United States Census Bureau. 2020. Waco City, Texas—QuickFacts. https://www.census.gov/quickfacts/ wacocitytexas. Zugegriffen: 23. Apr. 2021.

Van Gennep, Arnold. 1986. Übergangsriten. Frankfurt a.M., New York: Campus.

Venturi, Robert, Denise Scott Brown, und Steven Izenour. 2007. Lernen von Las Vegas: Zur Ikonographie und Architektursymbolik der Geschäftsstadt. Basel, Boston, Berlin: Birkhäuser.

Waco Tours. 2021. Our story. https://www.waco-tours.com/our-story. Zugegriffen: 23. Apr. 2021.

Wellman, James, Katie Corcoran, und Kate Stockly. 2020. High on god: how Megachurches won the heart of America. Oxford: Oxford University Press.

Wright, Lawrence. 2018. God save texas: a journey into the soul of the lone star state. New York: Knopf.

Wroten, Raquel. 2015. You ready to see your fixer upper? https://blogs.bible.org/you-ready-to-see-yourfixer-upper/. Zugegriffen: 14. Febr. 2021.

Zukin, Sharon. 1995. The cultures of cities. Malden: Blackwell. 